TKK Dissertations 43

Espoo 2006

LARGE-EDDY SIMULATION OF A ROUND JET IN A CROSS-FLOW

Doctoral Dissertation

Petri Majander
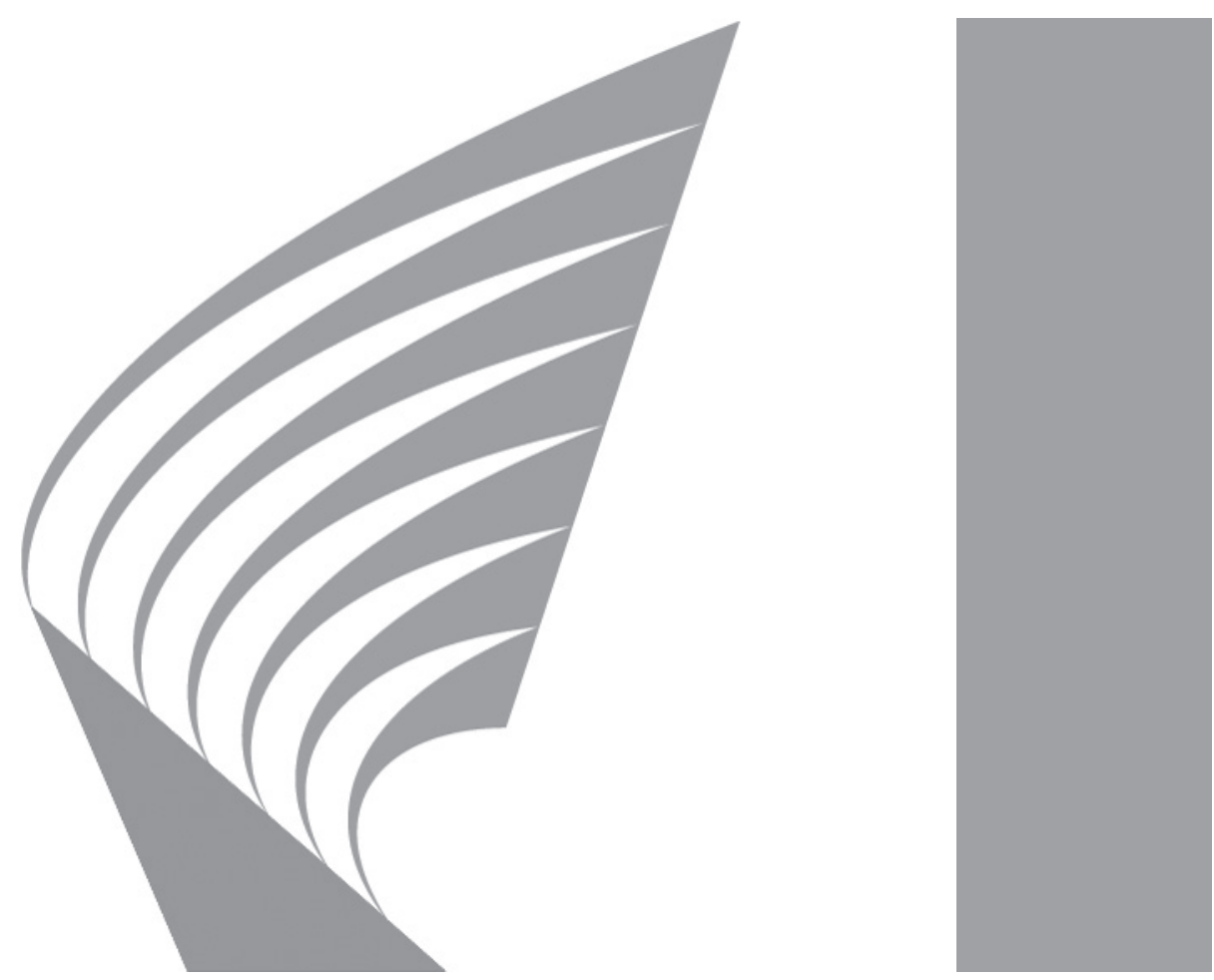

Helsinki University of Technology

Department of Mechanical Engineering

Laboratory of Applied Thermodynamics 
TKK Dissertations 43

Espoo 2006

\section{LARGE-EDDY SIMULATION OF A ROUND JET IN A CROSS-FLOW \\ Doctoral Dissertation}

\section{Petri Majander}

Dissertation for the degree of Doctor of Science in Technology to be presented with due permission of the Department of Mechanical Engineering for public examination and debate in Auditorium K216 at Helsinki University of Technology (Espoo, Finland) on the 1st of December, 2006, at 12 noon.

Helsinki University of Technology Department of Mechanical Engineering

Laboratory of Applied Thermodynamics

Teknillinen korkeakoulu

Konetekniikan osasto

Sovelletun termodynamiikan laboratorio 
Distribution:

Helsinki University of Technology

Department of Mechanical Engineering

Laboratory of Applied Thermodynamics

P.O. Box 4400

FI - 02015 TKK

FINLAND

URL: http://cfdthermo.tkk.fi/

Tel. +358-9-4511

E-mail: Petri.Majander@tkk.fi

(C) 2006 Petri Majander

ISBN-13 978-951-22-8404-7

ISBN-10 951-22-8404-9

ISBN-13 978-951-22-8405-4 (PDF)

ISBN-10 951-22-8405-7 (PDF)

ISSN 1795-2239

ISSN 1795-4584 (PDF)

URL: http://lib.tkk.fi/Diss/2006/isbn9512284057/

TKK-DISS-2186

Otamedia Oy

Espoo 2006 


\begin{tabular}{|c|c|c|c|}
\hline \multicolumn{2}{|c|}{$\begin{array}{l}\text { HELSINKI UNIVERSITY OF TECHNOLOGY } \\
\text { P. O. BOX 1000, FI-02015 TKK } \\
\text { http://www.tkk.fi }\end{array}$} & \multicolumn{2}{|c|}{ ABSTRACT OF DOCTORAL DISSERTATION } \\
\hline \multicolumn{4}{|c|}{ Petri Majander } \\
\hline \multicolumn{4}{|c|}{$\begin{array}{l}\text { Name of the dissertation } \\
\text { Large-eddy simulation of a round jet in a cross-flow }\end{array}$} \\
\hline Date of manuscript & 1.9 .2006 & Date of the dissert & 1.12 .2006 \\
\hline$\square$ Monograph & & $\mathrm{X}$ Article dissert: & tion (summary + original articles) \\
\hline $\begin{array}{l}\text { Department } \\
\text { Laboratory } \\
\text { Field of research } \\
\text { Opponent(s) } \\
\text { Supervisor } \\
\text { (Instructor) } \\
\end{array}$ & $\begin{array}{l}\text { Mechanical engineering } \\
\text { Applied thermodynamics } \\
\text { Computational fluid dynamics } \\
\text { Lars Davidson } \\
\text { Timo Siikonen } \\
\text { Timo Siikonen }\end{array}$ & & \\
\hline \multicolumn{4}{|l|}{ Abstract } \\
\hline \multicolumn{4}{|c|}{$\begin{array}{l}\text { Several aspects of large-eddy simulations (LES) are studied in this thesis. In the first part computational requirements } \\
\text { and methods are compared for the calculation of unsteady incompressible flow. Explicit time integration methods are } \\
\text { efficient especially in simple flow geometries and with low Reynolds numbers. An Adams-Bashford fractional } \\
\text { time-stepping scheme is used in the turbulent channel flow computations. }\end{array}$} \\
\hline \multicolumn{4}{|c|}{$\begin{array}{l}\text { The Smagorinsky model is a baseline subgrid-scale model used in LES. This and the dynamic version are assessed in } \\
\text { a turbulent channel flow. The models do not necessarily improve the results at a low Reynolds number if the } \\
\text { calculation is stabilized enough by the molecular or numerical viscous effects. With the second-order central scheme } \\
\text { the numerical error is estimated to be greater than the filtered stresses from the momentum equations. This estimate } \\
\text { alone suggests that there is no accurate model for instantaneous stresses for the low-order schemes. The main role of } \\
\text { the subgrid-scale model is to remove energy from the resolved scales and hence to stabilize the calculation. }\end{array}$} \\
\hline \multicolumn{4}{|c|}{$\begin{array}{l}\text { LES in any practical application, even at a low-Reynolds number, requires high computational resources. A parallel } \\
\text { solver based on a multi-block approach is written, where computational domain is divided between many structured } \\
\text { blocks. The blocks assigned to different processors communicate at the boundaries with an MPI standard. The solver } \\
\text { is tested with a turbulent cavity and a pipe flow. A linear speed-up and scale-up are achieved with equally balanced } \\
\text { processors loads. }\end{array}$} \\
\hline \multicolumn{4}{|c|}{$\begin{array}{l}\text { A large-eddy simulation of a round jet penetrating normally into a cross-flow is computed. The jet-to-cross-flow } \\
\text { velocity ratio is } 2.3 \text { at a Reynolds number of } 46700 \text {, based on the jet bulk velocity and the jet diameter. The } \\
\text { simulation is performed both with a steady and an unsteady boundary condition at the jet inlet pipe. A passive scalar } \\
\text { is discretized both with a central and a TVD discretization. The results are compared with each other and the } \\
\text { experimental measurements of Crabb, Durão and Whitelaw. The computation reproduced many phenomena present in } \\
\text { such a flow, like the shear layer ring vortices and a counter-rotating vortex pair. In general, a reasonable agreement } \\
\text { with the measurements was obtained. The unsteady boundary condition at the jet inlet increases the spreading of the } \\
\text { jet slightly. }\end{array}$} \\
\hline \multicolumn{4}{|c|}{ Keywords $\quad$ large-eddy simulation, jet in a cross-flow } \\
\hline ISBN (printed) & $951-22-8404-9$ & ISSN (printed) & $1795-2239$ \\
\hline \multicolumn{2}{|r|}{$951-22-8405-7$} & ISSN (pdf) & $1795-4584$ \\
\hline \multicolumn{2}{|l|}{ ISBN (others) } & Number of pages & 62 (summary) +120 (articles) \\
\hline \multicolumn{4}{|c|}{$\begin{array}{ll}\text { Publisher } & \text { TKK } \\
\end{array}$} \\
\hline \multicolumn{2}{|c|}{$\begin{array}{ll}\text { Print distribution } & \text { TKK }\end{array}$} & & \\
\hline \multicolumn{4}{|c|}{$\mathrm{X}$ The dissertation can be read at http://lib.tkk.fi/Diss/ } \\
\hline
\end{tabular}





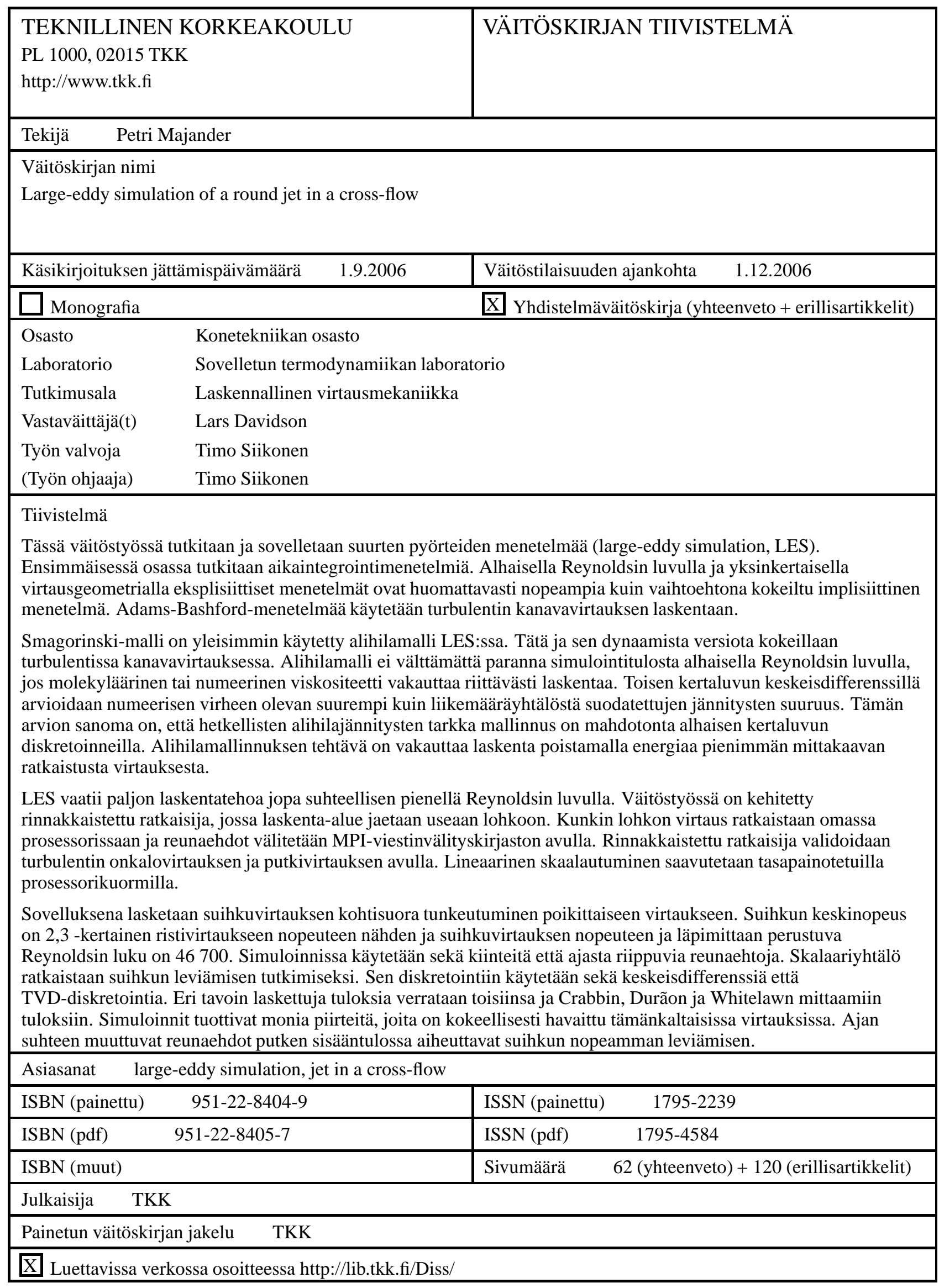





\section{Preface}

This work has been funded by the Academy of Finland, the Graduate School in Computational Fluid Dynamics, Tekes (Finnish Funding Agency of Technology and Innovation), Tekniikan edistämisäätiö and Imatran Voiman Säätiö. CSC (Center of Scientific Computing Ltd.) has provided me with the computer capacity. The contributions from the sources above are greatly acknowledged.

I thank Professor Timo Siikonen for instructing me not only in the research but in many issues more or less related to it. I thank all my former and present colleagues in the Laboratories of Applied Thermodynamics and Aerodynamics for all the help and fellowship I have had during this work at TKK. I have shared a room with Dr. Mizanur Rahman who has always been a very kind and helpful colleague. He has also introduced delicatessen from Bangladeshi cuisine to me. Dr. Antti Hellsten has readily given advice if needed and his work on a project management has eased also my burden. I am in gratitude to Mr. Juha-Veikko Ala-Juusela, Mrs. Tellervo Brandt, Mr. Ari Miettinen and Mr. Martti Alatalo for all the help and delightful discussions. I thank Professor Antti Oksanen from TUT for a pleasant co-operation.

My parents and my elder brother have provided me with a childhood environment that has nourished the mind of a researcher.

My wife Päivi and my children Martti, Mikko and Maria give the reason for everything.

Tiilää 1.9.2006

Petri Majander 


\section{Contents}

Preface $v$

Contents vii

$\begin{array}{ll}\text { List of Publications } & \text { ix }\end{array}$

1 Introduction 1

1.1 Turbulent Flow . . . . . . . . . . . . . . . . . . 1

1.2 Round Jet in a Cross-Flow . . . . . . . . . . . . . . . . 4

$\begin{array}{lll}2 & \text { Methods } & 7\end{array}$

2.1 Governing Equations . . . . . . . . . . . . . . 7

2.2 Conservation Form . . . . . . . . . . . . . . 10

2.3 Temporal and Spatial Discretizations . . . . . . . . . . . . 11

2.3.1 Explicit Euler Scheme . . . . . . . . . . . . 11

2.3.2 Fractional-Step Adams-Bashford Scheme . . . . . . . . 13

2.3.3 Implicit Scheme Based on SIMPLE. . . . . . . . . . . . 14

2.4 Pressure Coupling in SIMPLE . . . . . . . . . . . . 15

2.5 Calculation of Cell-Face Velocities . . . . . . . . . . 16

2.6 Total Variation Diminishing Scheme . . . . . . . . . . . 16

2.7 Numerical Test Filters _. . . . . . . . . . . . . . . . 17

2.8 Block Structure and Parallelization . . . . . . . . . . . 17

3 Computational Studies of Time Stepping 23

3.1 Motivation and Background . . . . . . . . . . . . . 23

3.2 Computations in Two Dimensions . . . . . . . . . . . 23

3.3 Conclusions . . . . . . . . . . . . . . . . . . 24

4 Studies of Subgrid-Scale Modelling $\quad 26$

4.1 Motivation and Background . . . . . . . . . . 26 
viii

4.2 Computations . . . . . . . . . . . . . . 26

4.3 Conclusions . . . . . . . . . . . . . . . . . 27

5 Parallel Solver $\quad 33$

5.1 Motivation and Background .................. 33

5.2 Test Computations . . . . . . . . . . . . . . 33

5.2 .1 Steady Cavity Flow . . . . . . . . . . . . 33

5.2 .2 Turbulent Cavity Flow . . . . . . . . . . . 34

5.2 .3 Fully Turbulent Pipe Flow . . . . . . . . . . . 35

5.2 .4 Conclusions ..................... 37

6 Jet in a Cross-Flow $\quad 38$

6.1 Flow Configuration ........................ 38

6.2 Results . . . . . . . . . . . . . . . . . 40

6.2 .1 Flow Field . . . . . . . . . . . . . . 40

6.2.2 Comparison to the Measurements . . . . . . . . . 43

6.3 Scalar Mixing . . . . . . . . . . . . . . 52

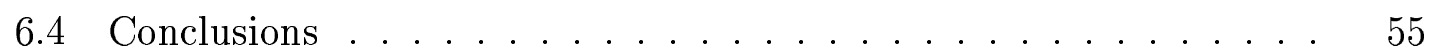

7 Concluding Remarks $\quad 56$

$\begin{array}{ll}\text { References } & 58\end{array}$

Errata 


\section{List of Publications}

This thesis consists of an overview and of the following publications which are referred to in the text by their Roman numerals.

I Majander P., Siikonen T., A comparison of time integration methods in an unsteady low-Reynolds-number flow, International Journal for Numerical Methods in Fluids, vol 39, pp 361-390, 2002.

II Majander P., Siikonen T., Evaluation of Smagorinsky-based subgrid-scale models in a finite-volume computation, International Journal for Numerical Methods in Fluids, vol 40, pp 735-774, 2002.

III Majander P., Siikonen T. , A parallel multi-block Navier-Stokes solver for large-eddy simulation in complex flows, 8th Finnish Mechanics Days, Espoo, Finland, pp. 395-406, June 12-13, 2003.

IV Majander P., Siikonen T., Large-Eddy Simulation of a Round Jet in a Crossflow, Proceedings of the 4th ECCOMAS Congress on Computational Methods in Applied Sciences and Engineering, July 24-28 2004, Jyvaskylä.

V Majander P., Siikonen T., Large-eddy simulation of a round jet in a crossflow, International Journal of Heat and Fluid Flow, vol 27, pp 402-415, 2006 . 


\section{Introduction}

\subsection{Turbulent Flow}

Fluid flows governed by the Navier-Stokes equations have been solved numerically since the early days of computers. The property of fluid flow that makes it a computational challenge is turbulence. Turbulence is not exactly mathematically defined and no analytical solutions for turbulent flows have been found. Hinze [13] gives the following definition: "Turbulent fluid motion is an irregular condition of flow in which the various quantities show a random variation with time and space coordinates, so that statistically distinct average values can be discerned."

Most researchers agree upon the following properties [13], [21], [38] :

- Turbulent flows are unsteady. The behaviour of the flow variables appears to be random if monitored as a function of time at a constant point and as a function of space at an instant moment of time.

- Turbulent flows are three-dimensional. Geophysical flows may possess twodimensional features in large scales, but the smallest scales are always threedimensional.

- Turbulent flows contain a lot of vorticity. The process of stretching the vorticity is important in increasing the intensity of turbulence.

- Turbulent flows are highly dissipative, viscous forces dissipate kinetic energy into heat. Unless friction from a wall or a speed difference sustains new energy into vortices, the turbulence will die.

- Turbulent flows contain a broad spectrum. The length scale of the largest eddies is of the order of the size of the flow geometry, the smallest scale is of the order of the viscous size, Kolmogorov length scale. The time scales behave correspondingly. 
- Turbulent flows mix much stronger than laminar flows. This is due to the vigorous motion of the fluid, which greatly enhances friction, heat and scalar transfer.

The direct solution of the Navier-Stokes equations is a very intensive task computationally and it has been performed mainly for academic purposes. Traditionally, turbulent flows have been computed with Reynolds-averaged Navier-Stokes (RANS) equations. The flow is averaged over a time to obtain a statistically steady flow. Another way of thinking about this process is to average a set of similar flows to produce an ensemble average.

In large-eddy simulation (LES), the large-scale motion is resolved and the small scales are modelled. This approach is based on the idea that the large-scale motion is highly dependent on boundary conditions, whereas small-scale motion is assumed to be relatively universal everywhere. The equations are filtered in space and the division into the large and small-scale motion is dependent on the length of the filter. LES has usually succeeded better than RANS modelling, especially in predicting separated flows. For most engineering computations, LES is too expensive today. Research has been done to combine RANS modelling and LES. This approach is called hybrid RANS/LES or detached eddy simulation (DES). In this approach RANS is used to model the flow in the near-wall region, which requires less resolution as compared to resolving boundary layer vortices with LES. Further away the LES is used to resolve the turbulence [7].

In turbulent flows, a wide range of scales is present. The largest scales are about the same magnitude as the geometry of the flow. In a flow over a cylinder, the largest vortices shed by the cylinder are of the same order as the diameter of the cylinder. In the boundary layer, the largest eddies are proportional to the thickness of the boundary layer. The dissipation rate at which the energy is cascaded into smaller vortices is the energy contained in large eddies per unit mass divided by their characteristic time scale [34]

$$
\epsilon \propto u^{2} / \frac{l}{u}=\frac{u^{3}}{l} .
$$


Here, $u$ is a characteristic root mean square value of the fluctuations and $l$ is the integral scale. The energy dissipated directly from the large eddies is obtained by dividing by the viscous time scale $u^{2} /\left(l^{2} / \nu\right)=\nu u^{2} / l^{2}$, which is small compared to $\epsilon$ in Eq. (1.1).

The smallest scales of motion adjust themselves to the value of the viscosity. As the small-scale motions have small time scales they are statistically quite independent of the mean flow and slower large-scale motion. With that assumption, the small-scale motion should depend only on the rate of the energy transfer from the large scales and on the kinematic viscosity. The following scales are referred to as Kolmogorov micro-scales of length $\eta$, time $\tau$ and velocity $v$,

$$
\begin{aligned}
\eta & \equiv\left(\nu^{3} / \epsilon\right)^{1 / 4} \\
\tau & \equiv(\nu / \epsilon)^{1 / 2} \\
v & \equiv(\nu \epsilon)^{1 / 4}
\end{aligned}
$$

The Reynolds number at the micro-scale level $\eta v / \nu$ is equal to one, which indicates that the small-scale motion is quite viscous. By substituting $\epsilon$ in Eq. (1.1) into Eqs. (1.2), the relations between the smallest and largest scales are obtained:

$$
\begin{aligned}
\eta / l & \equiv(u l / \nu)^{-3 / 4}=R e_{l}^{-3 / 4} \\
\tau / t & \equiv(u l / \nu)^{-1 / 2}=R e_{l}^{-1 / 2} \\
v / u & \equiv(u l / \nu)^{-1 / 4}=R e_{l}^{-1 / 4}
\end{aligned}
$$

As the Reynolds number increases, so does the gap between the smallest and the largest scales. Computational space must be discretized into a mesh where the mesh size $\Delta x$ in one direction $\propto R e_{l}^{-3 / 4}$. In a time-accurate solution, a time-step size should not exceed $\Delta x / u$. Suppose that to compute a turbulent flow in a box of size $l^{3}, \mathrm{~N}$ points $=(l / \Delta x)^{3}$ are required in space. In addition, the time to be computed is of the order of $l / u$. Hence, the total computational effort can be evaluated as $\propto\left(1 / R e_{l}^{-3 / 4}\right)^{4}=R e_{l}^{3}$. This strong dependence of the Reynolds number gives a quantified support to the statement in the beginning of this chapter: turbulence is a computational challenge. 


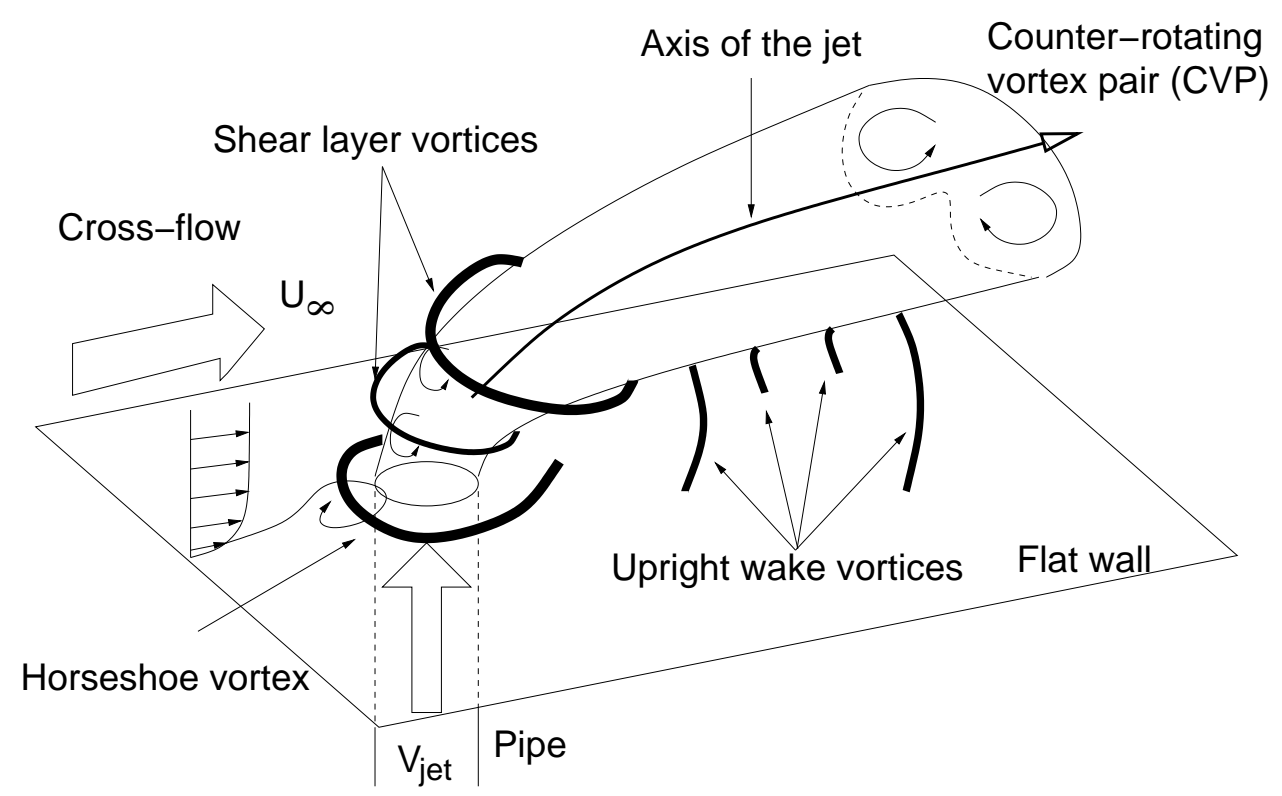

Figure 1.1: Schematic diagram showing the mean flow and the vortex systems of a jet in a cross-flow (adapted from [18] and [27]).

\subsection{Round Jet in a Cross-Flow}

Jets in a cross-flow are complex flows with many practical applications ranging from jets into combustors to V/STOL aircraft in transition flight. Perhaps the most familiar example in everyday life would be smoke rising from a chimney into a crosswind. The characteristics of a jet in a cross-flow are primarily dependent on the momentum flux from the jet exit to the momentum flux of the cross-flow over equal areas. When the jet and the cross-flow densities are equal, it is customary to define the jet-to-cross-flow velocity ratio

$$
J=\frac{V_{j e t}}{U_{\infty}}
$$

where $V_{j e t}$ is the bulk velocity from the orifice, averaged over time and the orifice area. $U_{\infty}$ is the free-stream velocity of the cross-flow. The mean flow pattern with typical instantaneous vortices is shown schematically in Fig. 1.1. The jet exits from the pipe normal to a cross-flow and is bent over. In the interaction of the colliding flows some typical structures are found. Ring vortices are created on the upstream 
side of the jet shear layer, which rolls up by a Kelvin-Helmholz-like instability. Near the wall there are horseshoe vortices just upstream of the jet. The counter-rotating vortex pair (CVP) is born at the jet exit and further downstream it becomes the dominant structure in the jet. Below the bent jet there is a system of upright wake vortices.

Due to the great practical relevance, many experimental, numerical and theoretical studies have been undertaken for jets in a cross-flow. Here some of them are cited. Keffer et al. [17] measured the trajectory of the jet and the velocity along it. The turbulent intensity was also measured along a line in the plane orthogonal to the jet trajectory. A more complete survey was conducted by Crabb et al. [6] who measured mean and fluctuating velocity magnitudes with a laser-Doppler anemometer near the jet exit and hot wires further downstream. The Reynolds number in their case was

$$
\operatorname{Re}_{D}=\frac{V_{j e t} D}{\nu}=46700
$$

where $V_{j e t}$ is the jet velocity and $D$ is the diameter of the pipe. The velocity ratio was either 1.15 or 2.3 , the latter case being the subject of a simulation in the present thesis. Also helium trace concentrations were measured in order to study the spreading of the jet. Andreopoulos and Rodi [1] used a triple wire probe to simultaneously measure all three components of velocity. The velocity ratios were $J=0.5,1$ and 2 with Reynolds numbers $R e_{D}=20500,41000$ and 82000 , respectively. Kelso et al. [18] studied the structure of round jets in cross-flows using flow visualization techniques and flying-hot-wire measurements. The jet-to-crossflow velocity ratios ranged from 2 to 4 and the Reynolds numbers varied between 440 and 6200 , based on the jet diameter and free-stream velocity. They studied various jet structures extensively. The concensus of opinion of many authors (eg. [1] and [18]) is that the CVP is formed by the shear layer vorticity emanating from the pipe flow. Kelso et al. described a roll-up mechanism of the jet shear layer that contributes to the CVP. Peterson and Plesniak [27] altered the direction of the channel flow to feed the jet through short holes into a cross-flow. If the channel is in the same direction as the cross-flow, a pair of vortices exists within the hole 
with the same sense of rotation as the CVP. A pair of vortices with the opposite sense of rotation existed as the channel flow was contrary to the cross-flow. The in-hole vortices interact with the CVP affecting the its strenght. They found that the weaker CVP has a lower trajectory and increased spanwise spreading.

Jets in a cross-flow have also been studied by simulations. Yuan et al. [40] performed LES at two jet-to-cross-flow velocity ratios, 2.0 and 3.3, and two Reynolds numbers, 1050 and 2100, based on the cross-flow velocity and the jet diameter. They discretized the computational area into a total of $1.34 \times 10^{6}$ control volumes. The jet in a cross-flow measured by Crabb et al. [6] was modelled with LES by Wille [39]. He used both a coarse and a fine mesh, which included 88440 and 997920 mesh points, respectively. Considering the Reynolds number of 46700 , based on the jet bulk velocity and the jet exit diameter, both grids are quite coarse even if the nearwall boundary layers were modelled. Wille does not explicitly state whether the Reynolds number is the same as in the experiment. Wegner et al. [37] studied turbulent mixing using LES. They varied the angle between the jet and the cross flow. The mixing was enhanced as the angle was increased, i.e. as the jet was directed against the cross-flow. The baseline flow in their simulation was that measured by Andreopoulos and Rodi [1].

In this work, large-eddy simulation is studied as a method, computational tools are constructed, and finally the method and the tools are applied to solve a jet in a cross-flow. An isocompressiple fluid model with constant properties for kinematic and molecular viscosity is applied. In the first chapter, the methods used in this work are presented (Papers I,II,III). In the second chapter, the computational issues concerning turbulence simulations are studied, in particular concerning the time advancement (Paper I). In large-eddy simulation a Smagorinsky model and its derivatives are the most used ones. In the third chapter, the effect of Smagorinskybased models on a simulation is assessed (Paper II). In the fourth chapter, a parallel solver is validated with some basic flow cases (Paper III). Finally, the solver is used to simulate a jet in a cross-flow with various parameters in the fifth chapter (Papers IV,V). The sixth chapter gives concluding remarks on the whole work. 


\section{Methods}

\subsection{Governing Equations}

The Navier-Stokes equations for incompressible flows with a passive scalar transport are written as

$$
\begin{aligned}
\frac{\partial u_{i}}{\partial x_{i}} & =0 \\
\frac{\partial u_{i}}{\partial t}+\frac{\partial u_{i} u_{j}}{\partial x_{j}} & =-\frac{1}{\rho} \frac{\partial p}{\partial x_{i}}+\frac{\partial}{\partial x_{j}}\left(\nu \frac{\partial u_{i}}{\partial x_{j}}\right) \\
\frac{\partial \theta}{\partial t}+\frac{\partial \theta u_{j}}{\partial x_{j}} & =\frac{\partial}{\partial x_{j}}\left(\alpha \frac{\partial \theta}{\partial x_{j}}\right),
\end{aligned}
$$

where $u_{i}$ is the velocity, $p$ is the pressure and $\nu$ is the kinematic viscosity. In the last equation $\theta$ is a scalar and $\alpha$ is molecular diffusivity. The molecular transport properties are considered to be constant in this work. The LES equations are formally derived by applying a filtering operation: a filtered variable is defined as

$$
\bar{f}(\mathbf{r}, t)=\int_{V} G\left(\left|\mathbf{r}-\mathbf{r}^{\prime}\right|\right) f\left(\mathbf{r}^{\prime}, t\right) d \mathbf{r}^{\prime}
$$

where $V$ is the volume of filtering and $G$ is the filter function. After filtering Eqs. (2.1) the LES equations take the following form

$$
\begin{aligned}
\frac{\partial \bar{u}_{i}}{\partial x_{i}} & =0 \\
\frac{\partial \bar{u}_{i}}{\partial t}+\frac{\partial \bar{u}_{i} \bar{u}_{j}}{\partial x_{j}} & =-\frac{1}{\rho} \frac{\partial \bar{p}}{\partial x_{i}}+\frac{\partial}{\partial x_{j}}\left(\nu 2 \bar{S}_{i j}-\tau_{i j}\right) \\
\frac{\partial \bar{\theta}}{\partial t}+\frac{\partial \bar{\theta} \bar{u}_{j}}{\partial x_{j}} & =\frac{\partial}{\partial x_{j}}\left(\alpha \frac{\partial \bar{\theta}}{\partial x_{j}}-q_{j}\right),
\end{aligned}
$$

where $\bar{u}_{i}$ are velocity components, $\bar{p}$ is pressure and $\rho$ is a constant density. The strain rate tensor is

$$
\bar{S}_{i j}=\frac{1}{2}\left(\frac{\partial \bar{u}_{i}}{\partial x_{j}}+\frac{\partial \bar{u}_{j}}{\partial x_{i}}\right) .
$$

The subgrid-scale stress and scalar flux are

$$
\begin{aligned}
\tau_{i j} & =\overline{u_{i} u_{j}}-\bar{u}_{i} \bar{u}_{j} \\
q_{j} & =\overline{\theta u_{j}}-\bar{\theta} \bar{u}_{j}
\end{aligned}
$$


respectively.

The Boussinesq approximation

$$
\tau_{i j}-\frac{\delta_{i j}}{3} \tau_{k k}=-2 \nu_{T} \bar{S}_{i j}=-\nu_{T}\left(\frac{\partial \bar{u}_{i}}{\partial x_{j}}+\frac{\partial \bar{u}_{j}}{\partial x_{i}}\right)
$$

relates SGS stresses $\tau_{i j}$ to the eddy viscosity $\nu_{T}$ and the resolved-scale strain rate tensor $\bar{S}_{i j}$. The Smagorinsky model [33] for the eddy viscosity is written as

$$
\nu_{T}=\left(C_{s} \Delta\right)^{2}|\bar{S}|
$$

where the parameter $C_{s}$ is called a Smagorinsky constant, $\Delta$ is a length scale and $|\bar{S}|=\sqrt{2 \bar{S}_{i j} \bar{S}_{i j}}$. The length scale represents the cell size and it is usually computed as $V^{1 / 3}$. A value of 0.18 for $C_{s}$ has been evaluated for the inertial range dynamics [21]. In the presence of a shear flow a smaller value must be used, which was first discovered by Deardorff [8]. He studied a channel flow and found that a smaller value for $C_{s}=0.1$ had to be used in the presence of a strong shear-driven turbulence. This observation has since then been confirmed by many studies. Furthermore, in the vicinity of the walls, the eddy viscosity must be reduced. This is usually carried out with the van Driest damping function. The subgrid-scale scalar flux can be modelled analogously with a mixing length gradient model

$$
q_{j}=-\frac{\nu_{s g s}}{P r_{s g s}} \frac{\partial \bar{\theta}}{\partial x_{j}}
$$

Depending on the flow, the molecular Prandtl number $\operatorname{Pr}$ and the direction and the distance of the scalar flux to the wall, values for the turbulent Prandtl number $\operatorname{Pr}_{\text {sgs }}$ ranging from 0.3 to 0.8 have been suggested [5].

Germano et al. [10] presented a new dynamic model, where the model coefficient is calculated during the simulation. This is based on the application of two different filters. In addition to the grid filter $G$, a test filter $\widehat{G}$ is applied. The test filter width $\widehat{\Delta}$ is larger than the grid filter width $\Delta$, usually $\widehat{\Delta}=2 \Delta$. The term grid filter implies that usually discretization takes care of the filtering in numerical computations without any explicit procedure. The grid filter and the test filter are applied to the momentum equations (2.1) to obtain the following equation:

$$
\frac{\partial \widehat{\bar{u}}_{i}}{\partial t}+\frac{\partial \hat{\bar{u}}_{i} \hat{\bar{u}}_{j}}{\partial x_{j}}=-\frac{1}{\rho} \frac{\partial \hat{\bar{p}}}{\partial x_{i}}-\frac{\partial T_{i j}}{\partial x_{i}}+\nu \frac{\partial^{2} \widehat{\bar{u}}_{i}}{\partial x_{j} \partial x_{j}},
$$


where the subtest stresses are given by

$$
T_{i j}=\widehat{\overline{u_{i} u_{j}}}-\hat{\bar{u}}_{i} \hat{\bar{u}}_{j}
$$

The test filter is now applied to the grid-filtered equations (2.5);

$$
\begin{gathered}
\frac{\partial \widehat{\bar{u}}_{i}}{\partial t}+\frac{\partial \widehat{\bar{u}}_{i} \widehat{\bar{u}}_{j}}{\partial x_{j}}=-\frac{1}{\rho} \frac{\partial \widehat{\bar{p}}}{\partial x_{i}}-\frac{\partial \widehat{\tau}_{i j}}{\partial x_{i}}-\frac{\partial \mathcal{L}_{i j}}{\partial x_{i}}+\nu \frac{\partial^{2} \widehat{\bar{u}}_{i}}{\partial x_{j} \partial x_{j}} \\
\mathcal{L}_{i j}={\widehat{\bar{u}_{i} \bar{u}_{j}}}-\hat{\bar{u}}_{i} \hat{\bar{u}}_{j} .
\end{gathered}
$$

Using Eqs. (2.12) and (2.14), the expression for $\mathcal{L}_{i j}$ can be written as

$$
\mathcal{L}_{i j}=T_{i j}-\hat{\tau}_{i j}
$$

Eqs. (2.13) represent the subtest-scale stresses whose length scale is less than the subtest filter width $\hat{\Delta}$. Eqs. (2.16) represent then the resolved turbulent stresses by the scales between the grid filter width $\Delta$ and the subtest-filter width $\hat{\Delta}$. Let us model both $\tau_{i j}$ and $T_{i j}$ by the same functional form of the Smagorinsky model:

$$
\begin{aligned}
\tau_{i j}-\frac{\delta_{i j}}{3} \tau_{k k} & =-2 C \Delta^{2}|\bar{S}| \bar{S}_{i j}=-2 C \beta_{i j}, \\
T_{i j}-\frac{\delta_{i j}}{3} T_{k k} & =-2 C \widehat{\Delta}^{2}|\widehat{\bar{S}}| \widehat{\bar{S}}_{i j}=-2 C \alpha_{i j} .
\end{aligned}
$$

Substitution of the stresses (2.17) and (2.18) into Eq. (2.16) with the assumption of a slow variation of $C(C(x, y, z, t) \approx \widehat{C}(x, y, z, t))$ gives

$$
\mathcal{L}_{i j}-\frac{\delta_{i j}}{3} \mathcal{L}_{k k}=-2 C\left(\widehat{\Delta}^{2}|\widehat{\bar{S}}| \widehat{\bar{S}}_{i j}-\Delta^{2} \mid \widehat{S}_{\bar{S}}\right) .
$$

In order to solve the set of equations for $C$ in Eqs. (2.19), Lilly [22] proposed solving Eqs. (2.19) in the least-square sense. The error

$$
Q=\left(\mathcal{L}_{i j}-\frac{\delta_{i j}}{3} \mathcal{L}_{k k}-2 C M_{i j}\right)^{2}
$$

where

$$
M_{i j}=\left(\widehat{\Delta}^{2}|\widehat{\bar{S}}| \widehat{\bar{S}}_{i j}-\Delta^{2} \mid \widehat{\bar{S}}_{i j}\right),
$$

is minimized by requiring $\partial Q / \partial C=0$, which gives

$$
C(x, y, z, t)=-\frac{\mathcal{L}_{i j} M_{i j}}{2 M_{i j} M_{i j}} .
$$


The numerator $\mathcal{L}_{i j} M_{i j}$ can have both positive and negative values. This indicates that the model can account for the backscatter of the turbulent energy, i.e., the energy is transferred from the small eddies to the large eddies. This happens in real flows locally at some instants of time, although the long time-average energy transport is from the large eddies to the small eddies. The non-positive viscosity hence produced tends to cause a numerical instability or even a singularity. In a turbulent channel flow, it is a common procedure to average the denominator and the numerator in homogeneous directions [10], [2], [26]

$$
C(y, t)=-\frac{\left\langle\mathcal{L}_{i j} M_{i j}\right\rangle_{x z}}{2\left\langle M_{i j} M_{i j}\right\rangle_{x z}} .
$$

In order to solve $C$ for complex geometries, Piomelli and Liu [28] developed the following localized dynamic model

$$
\mathcal{L}_{i j}-\frac{\delta_{i j}}{3} \mathcal{L}_{k k}=-2 C \alpha_{i j}+2 \widehat{C \beta_{i j}}
$$

where the coefficient $C$ under the filter is replaced with an estimate $C^{*}$, which is assumed to be known. Eqs. (2.24) can be solved by the contraction :

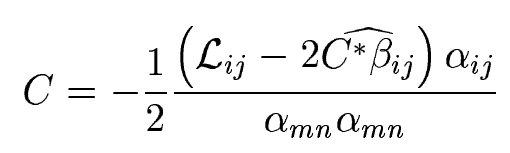

For $C^{*}$ one can use the value of the previous time step or iteration $C^{n-1}$. This model avoids some approximations of earlier models [22], [10]. An important feature is that the coefficient calculation is based on the local information. However, the values for $C$ must usually be limited in order to prevent destabilizing negative viscosity [28].

\subsection{Conservation Form}

The continuity equation in a conservation form becomes

$$
\int_{S} \rho \mathbf{V} \cdot d \mathbf{S}=0
$$

where $\rho$ is the density and $\mathbf{V}=u \mathbf{i}+v \mathbf{j}+w \mathbf{j}$ is the velocity of the fluid. The integration is taken over the control volume faces. This constraint simply states 
that at every moment the mass flow out from the control volume equals the mass flow in. The momentum equation in the $x$-direction is written as

$$
\begin{aligned}
\frac{\partial}{\partial t} \int_{V} \rho u d V & +\int_{S} \rho u \mathbf{V} \cdot \mathbf{n} d S+\int_{S} p n_{x} d S-\int_{V} \rho g_{x} d V \\
& -\int_{S} \mu\left(n_{x} \frac{\partial u}{\partial x}+n_{y} \frac{\partial u}{\partial y}+n_{z} \frac{\partial u}{\partial z}\right) d S=0
\end{aligned}
$$

Above, $p$ is the pressure, $\rho g_{x}$ is the body force and $\mathbf{n}=n_{x} \mathbf{i}+n_{y} \mathbf{j}+n_{z} \mathbf{k}$ is a unit normal of the control volume face. The equations in the $y$ - and $z$-directions are obtained by replacing the velocity component $u$ with $v$ and $w$ and the wall-normal component $n_{x}$ in the pressure term with $n_{y}$ and $n_{z}$, respectively. The scalar equation in a conservation form is written as

$$
\frac{\partial}{\partial t} \int_{V} \rho \theta d V+\int_{S} \rho \theta \mathbf{V} \cdot \mathbf{n} d S-\int_{S} \alpha\left(n_{x} \frac{\partial \theta}{\partial x}+n_{y} \frac{\partial \theta}{\partial y}+n_{z} \frac{\partial \theta}{\partial z}\right)=0
$$

\subsection{Temporal and Spatial Discretizations}

\subsubsection{Explicit Euler Scheme}

The simplest way to integrate an initial value differential equation is the explicit first-order accurate Euler method. The incompressible Navier-Stokes equations, Eqs. (2.27), are discretized as

$$
\rho \mathbf{V}_{i j k}^{n+1}=\rho \mathbf{V}_{i j k}^{n}-\frac{\Delta t}{V} \mathbf{R}_{i j k}^{n}-\frac{\Delta t}{V} \sum_{\text {faces }}(p \mathbf{S})^{n}
$$

where residual $\mathbf{R}_{i j k}=\left(R_{x} \mathbf{i}+R_{y} \mathbf{j}+R_{z} \mathbf{j}\right)_{i j k}$ is the net sum of convective and viscous fluxes surrounding the cell volume $i j k$

$$
R_{x}=\sum_{\phi=1}^{\text {faces }} S_{\phi} \rho u_{\phi} \mathbf{V}_{\phi} \cdot \mathbf{n}_{\phi}+S_{\phi}\left(\tau_{x j} n_{j}\right)_{\phi}=\sum_{\phi=1}^{\text {faces }} \dot{m}_{\phi} u_{\phi}+\mu_{\phi} S_{\phi}\left(n_{x} \frac{\partial u}{\partial x}+n_{y} \frac{\partial u}{\partial y}+n_{z} \frac{\partial u}{\partial z}\right)_{\phi} .
$$

Here, $\dot{m}_{\phi}=S_{\phi} \rho \mathbf{V}_{\phi} \cdot \mathbf{n}_{\phi}$ is a mass flow through the face $\phi$ and it is computed as an average between the neighbouring nodes. The velocity $u_{\phi}$ can be interpolated in many ways, but in LES a central difference is preferred, which implies that $u_{\phi}$ is interpolated as $\dot{m}_{\phi}$. In all the solvers used in this thesis the interpolation does not 
take into account the stretching of the mesh. In such meshes, a lower-order truncation error is present than the nominal truncation order suggests. The derivatives present in the viscous fluxes $\hat{F}_{\phi}^{v i s c}$ are calculated with the help of the generalized divergence theorem of Gauss. For a scalar $u$ the following equation applies

$$
\int_{V} \nabla u d V=\int_{S} u d \mathbf{S}
$$

Hence the derivative of $u$ in the $x$-direction is obtained from

$$
\left(\frac{\partial u}{\partial x}\right)_{i j k}=\frac{1}{V_{i j k}} \sum_{\phi=1}^{\text {faces }} S n_{x, \phi} u_{\phi} .
$$

The derivative at the face $\phi$ is obtained by integrating over a surrounding volume. In a uniform orthogonal mesh the interpolations correspond to a central difference. The Von Neumann method shows for a linear convection equation that the explicit Euler time advancement combined with the second-order centred scheme is unconditionally unstable. However, the addition of a diffusion term stabilizes the scheme.

The mass balance must be obeyed at both the old $(n)$ and the new $(n+1)$ time steps which can be used to derive the Poisson equation that relates pressure and the mass balance

$$
\begin{aligned}
& A_{W, i j k} p_{i-1, j k}+A_{E, i j k} p_{i+1, j k}+A_{S, i j k} p_{i, j-1, k}+A_{N, i j k} p_{i, j+1, k}+A_{B, i j k} p_{i j, k-1}+ \\
& A_{T, i j k} p_{i j, k+1}+A_{P, i j k} p_{i j k}=\sum_{\text {faces }}(\rho \mathbf{V} \cdot \mathbf{S})^{n}+\sum_{\text {faces }}(\mathbf{R} \cdot \mathbf{S})^{n},
\end{aligned}
$$

The coefficients and a more accurate derivation can be found in [23]. The computation is started with an initial guess. The residuals from the convective and viscous terms are computed and the right-hand side source of Eq. (2.33) is formed with the present mass balance included. The Poisson equation is iterated for pressures with a multigrid (MG) solver to a preset convergence limit. Now the time step $n$ is completed and the result can be written. Next, the new velocities at the time step $n+1$ are computed from the momentum equations. In Eq. (2.33), the first term on the right-hand side is the mass balance from the present time step $n$, which should be zero. This term can be retained to consider that, with an iterative method, the equation has not necessarily converged completely. 


\subsubsection{Fractional-Step Adams-Bashford Scheme}

Consider again Eqs. (2.27) with convection and diffusion terms discretized in the following second-order accurate scheme with respect to time. Pressure is discretized with a first-order accurate Euler scheme:

$$
\rho \mathbf{V}_{i j k}^{n+1}=\rho \mathbf{V}_{i j k}^{n}+\frac{\Delta t}{2 V_{i j k}}\left(3 \mathbf{R}_{i j k}^{n}-\mathbf{R}_{i j k}^{n-1}\right)-\frac{\Delta t}{V_{i j k}} \sum_{\text {faces }}(p \mathbf{S})^{n+1}
$$

where $\mathbf{R}_{i j k}$ is defined by Eq. (2.30), although the sum is taken at the faces around the control volume $i j k$. This method is called the Adams-Bashford ${ }^{1}$ two-step scheme and it is the simplest multistep method (see e.g. [4]). To compute Eq. (2.34) a fractionalstep approach presented by Kim and Moin [19] is applied. The computation is advanced in two parts:

$$
\begin{aligned}
\rho \mathbf{V}_{i j k}^{*} & =\rho \mathbf{V}_{i j k}^{n}+\frac{\Delta t}{2 V_{i j k}}\left(3 \mathbf{R}_{i j k}^{n}-\mathbf{R}_{i j k}^{n-1}\right) \\
\rho \mathbf{V}_{i j k}^{n+1} & =\rho \mathbf{V}_{i j k}^{*}-\frac{\Delta t}{V_{i j k}} \sum_{\text {faces }}(p \mathbf{S})^{n+1} .
\end{aligned}
$$

For the latter equation, the mass balance is required for the control volume $V_{i j k}$. By performing the surface integral over the faces of the volume, a Poisson equation for the pressure is obtained:

$$
\begin{aligned}
& A_{W, i j k} p_{i-1 k}+A_{E, i j k} p_{i+1 j k}+A_{S, i j k} p_{i j-1 k}+A_{N, i j k} p_{i j+1 k}+ \\
& A_{B, i j k} p_{i j k-1}+A_{T, i j k} p_{i j k+1}+A_{P, i j} p_{i j k}=\sum_{\text {faces }}\left(\rho \mathbf{V}^{*} \cdot \mathbf{S}\right)
\end{aligned}
$$

where the coefficients are same as with the Euler scheme in Eq. (2.33).

The computation is started with an initial guess which is used for both previous steps. Intermediate velocities $\mathbf{V}^{*}$ are computed and interpolated to the cell faces to calculate the mass balance. The Poisson equation (2.37) is iterated for the pressures with a multigrid (MG) solver to a preset convergence limit. Finally, the new velocities can be corrected from Eq. (2.36) to obtain the result at the new time step.

\footnotetext{
${ }^{1}$ Another spelling of the name found in literature is Bashforth.
} 


\subsubsection{Implicit Scheme Based on SIMPLE.}

Let us consider the following generic multistep method

$$
\begin{aligned}
\frac{V_{i j k}}{\Delta t}\left[(1+\gamma)(\rho \mathbf{V})^{n+1}\right. & \left.-(1+2 \gamma)(\rho \mathbf{V})^{n}+\gamma(\rho \mathbf{V})^{n-1}\right] \\
& =(1-\beta) \mathbf{R}^{n}+\beta \mathbf{R}^{n+1}
\end{aligned}
$$

where residual $\mathbf{R}$ also includes the pressure term. With different choices of parameters $\beta$ and $\gamma$, the following time advancement schemes can be realized, with $\beta=$ $\gamma=0$ the explicit Euler, with $\beta=1, \gamma=0$ an implicit Euler, with $\beta=0.5, \gamma=$ 0 a Crank-Nicolson and with $\beta=1.0, \gamma=0.5$ a three-level implicit (3-LI) scheme. The last two methods are second-order accurate. Equation (2.38) is linearized at the state $k$ between the solved state $n$ and the state $n+1$ to be computed:

$$
\begin{aligned}
\frac{V_{i j k}}{\Delta t}((1 & \left.+\gamma) \rho\left(\mathbf{V}^{k}+\mathbf{V}^{n+1}-\mathbf{V}^{k}\right)-(1+2 \gamma) \rho \mathbf{V}^{n}+\gamma \rho \mathbf{V}^{n-1}\right) \\
& =(1-\beta) \mathbf{R}^{n}+\beta\left(\mathbf{R}^{k}+\left[\frac{\partial \mathbf{R}}{\partial \mathbf{V}}\right]^{k}\left(\mathbf{V}^{n+1}-\mathbf{V}^{k}\right)\right)
\end{aligned}
$$

Let us write $\Delta \mathbf{V}=\mathbf{V}^{n+1}-\mathbf{V}^{k}$ and regroup the terms as follows:

$$
\begin{aligned}
& \left(\frac{V}{\Delta t}(1+\gamma)-\beta\left[\frac{\partial \mathbf{R}}{\partial \mathbf{V}}\right]^{k}\right) \Delta \mathbf{V} \\
= & \frac{V}{\Delta t}\left(-(1+\gamma) \mathbf{V}^{k}+(1+2 \gamma) \mathbf{V}^{n}-\gamma \mathbf{V}^{n-1}\right)+(1-\beta) \mathbf{R}^{n}+\beta \mathbf{R}^{k}
\end{aligned}
$$

From Eq. (2.40), $\mathbf{V}^{n+1}$ can be iterated. The scheme can be stabilized by using a local time step. This can be done by replacing $\gamma$ on the diagonal by $\Delta t / \Delta \Theta$ where $\Delta \Theta$ is the local time step of the steady-state solver [15]. Both constant and local time steps have been applied in this thesis. Equations (2.40) are solved sequentially within a time step. In linearization a first-order upwind scheme is used for the convective part and simplified viscous flux to avoid a large molecule.

After linearization, the equation obtained for the velocity increment $\Delta u$ is

$$
\begin{gathered}
\sum_{n b} A_{n b} \Delta u_{n b}=-\sum_{\phi=1}^{f a c e s}\left(S n_{x}\right)_{\phi} \Delta p_{\phi}^{k} \\
+\frac{V}{\Delta t}\left(-(1+\gamma) u^{k}+(1+2 \gamma) u^{n}-\gamma u^{n-1}\right)+\left[(1-\beta) R_{x}^{n}+\beta R_{x}^{k}\right]
\end{gathered}
$$


where the sum on the left-hand side is over the neighbour nodes. In $\Delta u=u_{i j k}^{n+1}-u_{i j k}^{k}$ the state $k$ lies between the solved state $n$ and the state $n+1$ to be computed. The first term on the right-hand side includes the pressure at the time level $n+1$ and in an iterative solution the term is ignored. The coefficients and a more accurate derivation can be found in [23].

\subsection{Pressure Coupling in SIMPLE}

The solution of the momentum equation must be coupled with pressure. The last two terms of Eq. (2.41) cancel when the iteration is converged. The linearized increments $\Delta u$ and $\Delta p$ are replaced in the following by iterative corrections $u^{\prime}$ and $p^{\prime}:$

$$
\sum_{n b} A_{n b} u_{n b}^{\prime}=-\sum_{\phi=1}^{\text {faces }} S_{\phi} n_{\phi x} p_{\phi}^{\prime},
$$

where the standard simplification utilized in the SIMPLE method is to drop the non-diagonal terms from Eq. (2.42) to derive a manageable equation. The continuity equation states that

$$
\begin{aligned}
& \sum_{\phi=1}^{\text {faces }_{i j}}\left(\rho\left(\mathbf{V}^{*}+\mathbf{V}^{\prime}\right) \cdot \mathbf{S}\right)_{\phi}=0 \\
& \sum_{\phi=1}^{\text {faces }_{i j}}\left(u^{\prime} n_{x} S\right)_{\phi}+\left(v^{\prime} n_{y} S\right)_{\phi}+\left(w^{\prime} n_{z} S\right)_{\phi}=-\sum_{\phi=1}^{\text {faces }_{i j}}\left(\rho \mathbf{V}^{*} \cdot \mathbf{S}\right)_{\phi}
\end{aligned}
$$

where $\mathbf{V}^{*}$ is the velocity field that does not obey the mass balance and $\mathbf{V}^{\prime}$ is the iterative correction.

After some assumptions and manipulations of the terms, we finally obtain the Poisson equation for the pressure corrections

$$
\begin{aligned}
& B_{P, i j k} p_{i j k}^{\prime}+B_{W, i j k} p_{i-1, j k}^{\prime}+B_{E, i j k} p_{i+1, j k}^{\prime}+B_{S, i j k} p_{i, j-1, k}^{\prime}+B_{N, i j k} p_{i, j+1, k}^{\prime}+ \\
& B_{B, i j k} p_{i j k-1}^{\prime}+B_{T, i j k} p_{i j k+1}^{\prime}=\Delta \dot{m}_{i j k}
\end{aligned}
$$

where the coefficients can be found in [23]. 


\subsection{Calculation of Cell-Face Velocities}

In a co-located, orthogonal grid, the velocity does not depend on the pressure at the same node $(i j k)$, which can lead to a non-physical converged solution, a phenomenon known as a checkerboard solution. Therefore, methods have been developed to couple the pressure and the velocity in the calculation of mass balance. Rhie and Chow [30] have presented a remedy for uncoupling, which is in wide use. Johansson and Davidson [16] derived a simplified method that is used in the present SIMPLE solvers. In the calculation of mass balance, the cell-face velocities are computed as

$$
\bar{u}_{i-\frac{1}{2}, j k}=\frac{1}{2}\left(\bar{u}_{i-1, j k}+\bar{u}_{i j k}\right)+C \frac{S_{i-\frac{1}{2}, j k}}{4 A_{P, i-\frac{1}{2}, j k}}\left[p_{i-2, j k}-3 p_{i-1, j k}+3 p_{i j k}-p_{i+1, j k}\right] .
$$

The pressure term in Eq. (2.45) adds numerical dissipation. In a smooth pressure field, the term will become negligible. The pressure term is multiplied by a constant $C$ in the range $0.01-0.5$ to adjust an optimum coupling [24].

\subsection{Total Variation Diminishing Scheme}

The central difference leads easily into spurious wiggles in the scalar field. In the mean mixing fraction the values below zero and over one are present. The Total Variation Diminishing (TVD) scheme reduces the overshoots effectively. Here, a TVD scheme is implemented with a minmod limiter combined into a Monotone Upwind-Centered Scheme for Conservation Law (MUSCL) [14]. With the central

difference discretization, the variables extrapolated at the cell face $i+\frac{1}{2}$ from the left- and right-hand side are

$$
\begin{aligned}
& \theta_{i+\frac{1}{2}}^{L}=\theta_{i}+\frac{1}{2} \phi\left(r_{i+\frac{1}{2}}^{-}\right)\left(\theta_{i+1}-\theta_{i}\right) \\
& \theta_{i+\frac{1}{2}}^{R}=\theta_{i+1}-\frac{1}{2} \phi\left(r_{i+\frac{1}{2}}^{+}\right)\left(\theta_{i+1}-\theta_{i}\right)
\end{aligned}
$$

where the minmod limiter is defined as

$$
\begin{array}{r}
\phi(r)=\min (r, 1) \quad, \quad r>0 \\
\phi(r)=0 \quad, \quad r \leq 0
\end{array}
$$


and the arguments of the limiter are the ratios of the consequent variables

$$
r_{i+\frac{1}{2}}^{-}=\frac{\theta_{i}-\theta_{i-1}}{\theta_{i+1}-\theta_{i}} \quad r_{i+\frac{1}{2}}^{+}=\frac{\theta_{i+2}-\theta_{i+1}}{\theta_{i+1}-\theta_{i}}
$$

If the limiter is activated the discretization becomes upwind-biased and numerical dissipation is introduced.

\subsection{Numerical Test Filters}

The filtering operation in one dimension over equidistant points $a, b$ and $c$ is defined as an integral

$$
\overline{f(b)}=\int_{a}^{c} G\left(b-x^{\prime}\right) f\left(x^{\prime}\right) d x^{\prime} .
$$

The top-hat filter is then

$$
\begin{array}{llll}
G(b-x)=\frac{1}{c-a} & \text {,if } & |b-x| \leq \frac{c-a}{2} \\
G(b-x)=0 & \text {,if } & |b-x|>\frac{c-a}{2} .
\end{array}
$$

Let us consider a numerical integration using three points $a, b$ and $c$. The trapezoidal rule leads to the following second-order accurate trapezoidal filter [4]

$$
\overline{f(b)}=\frac{1}{4}[f(a)+2 f(b)+f(c)] .
$$

Application of the fifth-order accurate Simpson quadrature leads to the following fourth-order filter [4]

$$
\overline{f(b)}=\frac{1}{6}[f(a)+4 f(b)+f(c)]
$$

In two and three dimensions, the filters can be applied in series in each dimension. The three-dimensional filters thus obtained are illustrated in Fig. 2.1.

\subsection{Block Structure and Parallelization}

A computational grid may be difficult to define on complex geometries by utilizing a structured grid. A well-known remedy for this is a multi-block structure, where the computational domain is divided between many structured blocks that are connected together [15], [31]. Ghost cells are used to set the boundary condition. Two 
layers of cells are defined to ensure a second-order accuracy, if upwind-biased discretizations are used, and for the Rhie and Chow interpolation. The cell values at the two layers at the edge of the block are transferred to the ghost cells of the neighbouring block and vice versa. The neighbouring blocks may have a different orientation. The connective boundary condition applies an orientation matrix and an offset vector at each connective face. These relate the indices in the boundary data transfer, as illustrated in Fig. 2.2. In the current version of the solver, each face must connect completely to another face and the grid lines must be continuous. The parallelization is based on dividing the blocks among different processors, although many blocks can be assigned into one processor. The parallelization is implemented with a standard called Message-Passing Interface (MPI) [12]. Each block reserves a memory space which is used to transmit the boundary data with MPI_SEND and MPI_RECV operations. These operations are blocking; that is, the control does not return to the user program until the message has been received. Therefore, the order of communication between the processes is solved before the computation is started. Each message sent must be received in the proper order, otherwise the computation deadlocks. The grid should be divided as equally as possible between different processors in order to provide a good balancing. All processes compute the problem equally but only the master process reads in an input file, a boundary data file and the grid. The master defines the boundary data needed and computes the order of the communication. The master transmits the data by an MPI_BCAST command to all workers and the workers' part of the grid by an MPI_SEND command. A flow chart of the solver is presented in Fig. (2.3). After reading the input data and the mesh, each process computes the local memory size needed and allocates a one-dimensional table that contains all the flow variables. No memory space is wasted, even if the blocks differ in size within one process or between processes.

The computation is started with an initial guess, which is also copied at the previous time levels. The momentum equations (2.41) are solved in series with an algebraic multigrid solver. The mass balance is calculated with face velocities (2.45) 
Trapezoidal filter
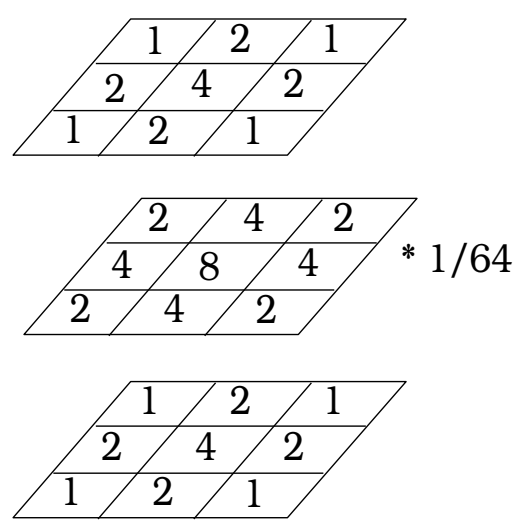

Simpson filter
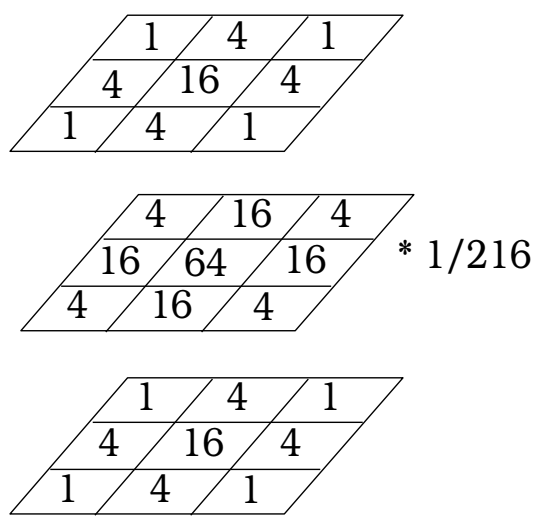

Figure 2.1: Trapezoidal and Simpson filters in three dimensions.

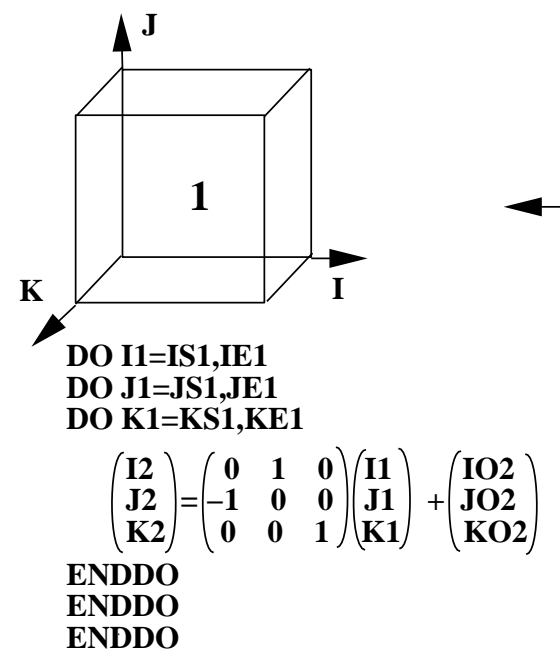

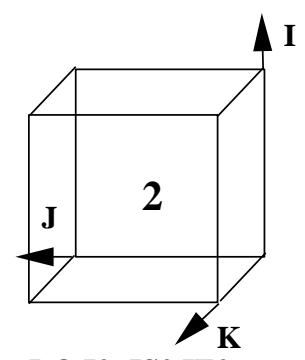

DO I2=IS2,IE2

DO J2=JS2,JE2

DO K2=KS2,KE2

$\left(\begin{array}{l}\mathbf{I} 1 \\ \mathbf{J} 1 \\ \mathrm{~K} 1\end{array}\right)=\left(\begin{array}{ccc}0 & -1 & 0 \\ 1 & 0 & 0 \\ 0 & 0 & 1\end{array}\right)\left(\begin{array}{l}\mathbf{I} 2 \\ \mathbf{J} 2 \\ \mathrm{~K} 2\end{array}\right)+\left(\begin{array}{l}\mathbf{I O 1} \\ \text { JO1 } \\ \text { KO1 }\end{array}\right)$

ENDDO

ENDDO

ENDDO

Figure 2.2: The connective boundary condition takes into account a different orientation between the blocks. An orientation matrix and an offset vector relates the indices between the blocks. 


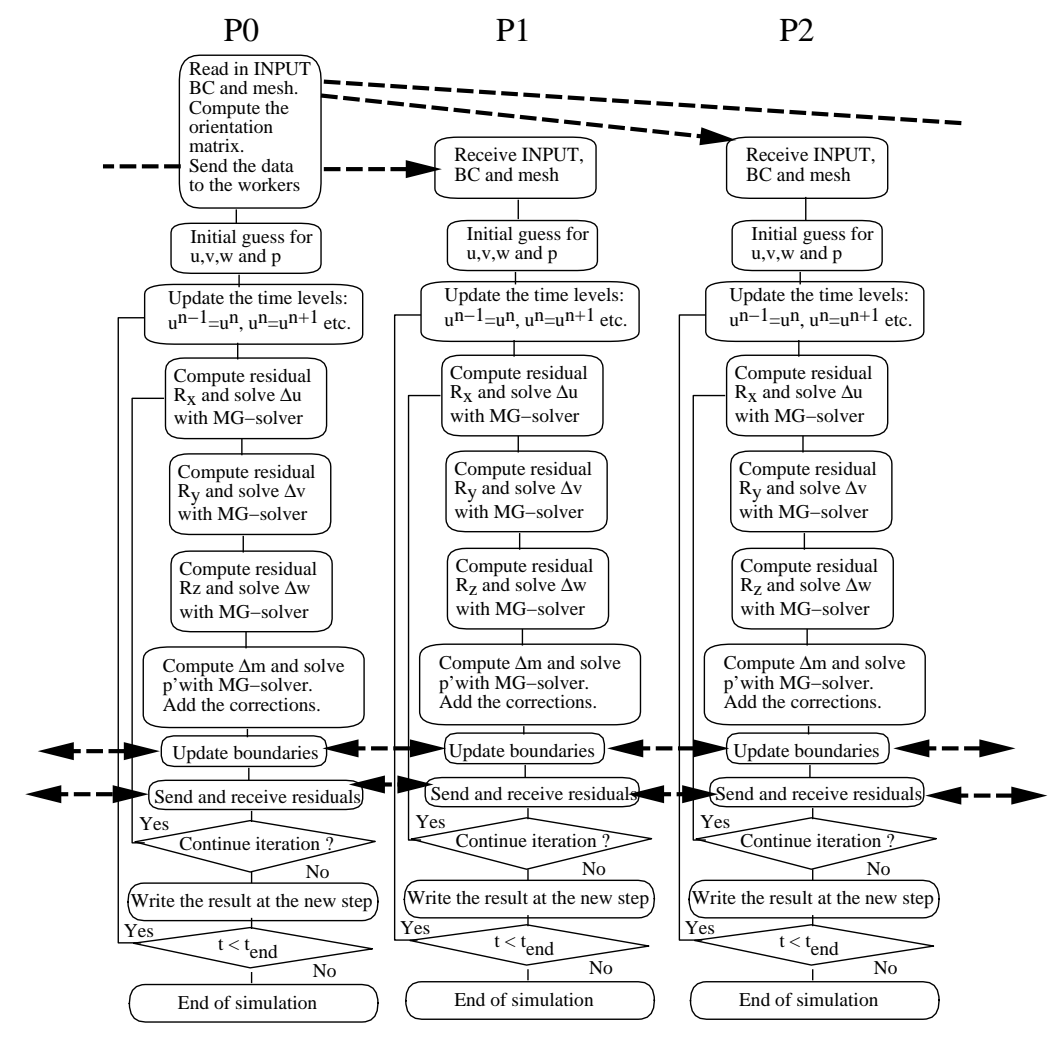

Figure 2.3: A flow diagram of the parallel code. The dashed arrows represent communication between the processors.

that are coupled with the pressure. The mass flux error is used as a source in the pressure correction equation (2.44), which is solved with a multigrid (MG) solver. The MG algorithm solves the problem at the dense level first and moves the residual to the next coarsest level as a source. The coarse grid problem is approximated from the finer one. The process is called the Galerkin Coarse-Grid Approximation (GCA). After the coarsest grid level, the corrections are added to the unknown of each finer level, or the problem can be iterated also on the way up. The line Gauss Seidel (LGS) method is used at each level as a smoother. Reference [24] and Pensala (unpublished memorandum ' 3 -D multigrid solver for Poisson-type equations' in Finnish, HUT, 1996) give a detailed description of the MG solver. The pressure corrections $p^{\prime}$ and the velocity corrections are added to the pressures and the velocities multiplied by 
the under-relaxation coefficients $\alpha_{p}$ and $\alpha_{u}$, respectively. Depending on the case, $\alpha_{p}$ usually varies from 0.1 to 0.8 . In order for the velocities to satisfy the continuity equation, $\alpha_{u}$ should be 1 at this stage.

The implicit momentum equations and the Poisson equations for the pressure are solved independently in all blocks. During the iteration, a Diriclet condition $\left(\Delta \mathbf{V}, p^{\prime}=0\right)$ is set at the first ghost cell of the connective boundaries. After each sub-iteration, cycle the boundary condition is updated for velocities and pressure $(\mathbf{V}, p)$. The boundary value is lagging in time which is solved by iterating within a time step. Let us study a particular problem in a two-dimensional situation where four blocks meet at a corner in Fig. 2.4. After one exchange of boundary data the value of the ghost corner cells is not correct. Rizzi et al. [31] have shown that, by repeating the same boundary data transfer twice, the values at the corners are also correct. In three dimensions the transfer must be repeated three times. However, since the message passing between processes consumes time the boundary values are transfered only once after each iteration cycle. Owing to the sub-iterations, also a ghost corner value is correct in a converged solution. In a similar way to that of Bui [3], all the boundary values at the connecting boundary are copied in a single vector that is transmitted with a single message passing call. The criterion of convergence can be set in many different ways and it can depend on any primary variable or the balance of mass fluxes. Usually, an $L_{1}$ - or $L_{2}$-norm of a residual of some variable must converge below a preset limit. As the convergence is reached, the solution of the new time level is started by updating the variables at the old and present time levels. 
a)

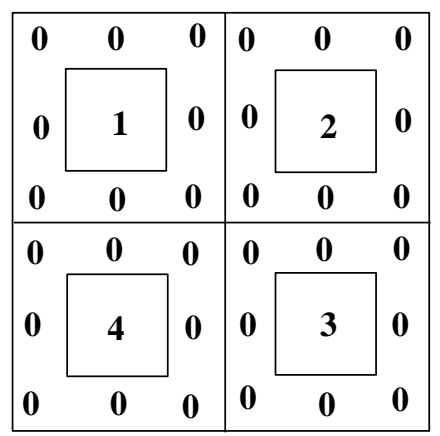

b)

\begin{tabular}{|c|c|c|c|c|c|}
\hline $\mathbf{0}$ & 0 & 0 & 0 & 0 & 0 \\
\hline 0 & 1 & 2 & 1 & 2 & 0 \\
\hline 0 & 4 & 0 & 0 & 3 & 0 \\
\hline 0 & 1 & 0 & 0 & 2 & 0 \\
\hline 0 & 4 & 3 & 4 & 3 & $\mathbf{0}$ \\
\hline 0 & 0 & 0 & 0 & 0 & 0 \\
\hline
\end{tabular}

\begin{tabular}{|c|c|c|c|c|c|}
\hline 0 & 0 & 0 & 0 & 0 & $\mathbf{0}$ \\
\hline 0 & 1 & 2 & 1 & 2 & $\mathbf{0}$ \\
\hline $\mathbf{0}$ & 4 & 3 & 4 & 3 & $\mathbf{0}$ \\
\hline 0 & 1 & 2 & 1 & 2 & $\mathbf{0}$ \\
\hline 0 & 4 & 3 & 4 & 3 & 0 \\
\hline 0 & ( & 0 & ( & 0 & 0 \\
\hline
\end{tabular}

Figure 2.4: One exchange of the boundary value does not set correct values in the corner ghost cells where four blocks meet (b). By repeating the transfer the values in the corner ghost cells will be updated [31] (c). In three dimensions three successive transfers are needed. 


\section{Computational Studies of Time Stepping}

\subsection{Motivation and Background}

A stability analysis of a linear convection equation for an explicit Euler scheme with a first-order upwind discretization reveals that a longest possible time step is limited by the Courant-Friedrics-Lax condition

$$
C F L=\frac{U \Delta t}{\Delta x} \leq 1
$$

In general, the limit condition above applies to all explicit methods excluding some methods like Runge-Kutta, which may violate it by a factor of two or three. In a simple flow case like a channel flow, this poses no restriction for a time accurate solution. However, a complex geometry involves many parts and blocks (in structured grids) with clustering of cells near solid walls. This will probably limit the time-step size unnecessarilly. Violating the CFL condition somewhere in the domain may not deteriorate the overall solution but savings in the computational times can be gained. The stability of the implicit schemes is also an advantage. It should be mentioned that the von Neumann stability analysis of a linear convection equation for the explicit Euler scheme with the second-order central discretization reveals that such method is uncontionally unstable. However, the addition of a diffusion term stabilizes the scheme.

\subsection{Computations in Two Dimensions}

Explicit Euler and Adams-Bashford methods are compared with an iterative implicit three-level method based on a SIMPLE solver in Paper I. A two-dimensional vortexshedding flow over a cylinder is the test case. Reynolds numbers at a low-range of 100 - 1600 are used. The grid clustering near the surface depends on the Reynolds number. The time-step size is varied and its effect on the results is studied. Finally, a high-Reynolds number case of a biconvex airfoil $\left(R e=1.83 \times 10^{6}\right)$ is calculated. All the calculations are two-dimensional although the real flow would be three- 
dimensional from $\operatorname{Re}=400$ to highly turbulent airfoil. The purpose of the twodimensional simulations is to compare solvers with reasonable computational cost.

\subsection{Conclusions}

The implicit method allows the time step to be lengthened beyond $C F L=1$. The number of iterations within the time step also grows in order to achive the same convergence and accuracy. At $R e=100$ the explicit methods are five times faster than the implicit ones with the largest time step. At the higher Reynolds number, $R e=1600$, the factor is four. The maximum local $C F L$ number with this time step is approximately 30 at $R e=1600$, so the step is too long to capture the fine structure in a turbulent flow. As the step size is decreased by one decade, the explicit methods are faster by a factor of ten at $R e=100$. At the Reynolds number of 1600 , the factor is slightly lowered to eight. The results obtained with the Reynolds numbers of 400 and higher deviate from the measured due to the three-dimensionality of the real flow. However, this hardly affects the comparison between the methods used and their computational efficiency.

At the high-Reynolds number $R e=1.83 \times 10^{6}$ the computational mesh includes small cells near the solid boundaries that restrict the time-step length. An implicit method is able to use a time-step size that is about 20 times larger than that of an explicit one. The force coefficients and the statistically gathered first and second moments are practically identical from both calculations. Owing to the subiterations and the solution of the implicit momentum equations, the computational time is still slightly more than that of the explicit calculation. The difference is negligible compared to the low-Reynolds number cases, however.

The implicit method is based on the SIMPLE algorithm, which can possibly be improved by different derivative schemes like SIMPLEC or PISO. Another important issue, which has been omitted in this study, is parallelization. In practice, most turbulent time-dependent cases are computationally highly intensive tasks which are solved in parallel systems. A common approach is to divide the physical solution 
domain into subdomains and assign each subdomain to one processor. The amount and frequency of data exchanged between processors affects the performance. For instance, the solution of the elliptic Poisson equation might be an intensive task in terms of data exchange, if solved in a subdomain parallelization scheme. The effect on the performance may differ depending on the computational algorithm used. 


\section{Studies of Subgrid-Scale Modelling}

\subsection{Motivation and Background}

Najjar and Tafti [26] studied the discrete test filters at a low Reynolds number $R e_{\tau}$ $=180$. They found that a high-order test filter produced a lower turbulent viscosity than a top-hat test filter. This dynamic procedure also sensed the dissipative upwind-biased scheme and adjusted the dynamic constant accordingly. However, in their conclusion they were not convinced that inclusion of the SGS model would always produce better results in finite-difference approximations. The reason for this is due to numerical errors rather than due to the model itself, they concluded. Najjar and Tafti [25] studied the effect of the grid resolution in the channel flow simulation at a higher Reynolds number, $R e_{\tau}=1050$. The coarse grid simulation predicted too low wall stresses and the core velocities were too high. They concluded that a coarse grid could not capture the energy-producing near-wall structures. The application of the test filter in all the directions (i.e. also in the inhomogeneous direction) decreased the SGS stresses. Sagaut et al. [32] computed the channel flow with various self-adaptive SGS models combined with a finite-difference scheme at $R e_{\tau}=180$ and $R e_{\tau}=400$. A general trend was that the addition of the SGS models decreased the wall stress.

\subsection{Computations}

In Paper II a fully turbulent channel flow at Reynolds numbers $R e_{b}=2800$ and $R e_{b}=12500$ (based on the bulk velocity and half the channel height) is the test case in the study of large-eddy simulation with a co-located finite-volume technique. The mass flow is kept constant in the simulations by adjusting the average pressure gradient separated from that pressure solved from the Poisson equation. The results from simulations with the constant and the dynamic Smagorinsky SGS models are compared to a non-modelled simulation. The effect of the grid resolution on the results is studied with and without the SGS model. At $R e=2800$ calculations are 
Table 4.1: The parameters of the grids used .

\begin{tabular}{|c|ccc|cccc|}
\hline Grid & $N_{x}$ & $N_{y}$ & $N_{z}$ & $\Delta x^{+}$ & $\Delta y_{\min }^{+}$ & $\Delta y_{\max }^{+}$ & $\Delta z^{+}$ \\
\hline coarse & 16 & 64 & 16 & 71 & 1.0 & 16.8 & 36 \\
medium & 32 & 64 & 32 & 36 & 1.0 & 16.8 & 18 \\
fine & 64 & 64 & 64 & 18 & 1.0 & 16.8 & 9 \\
\hline
\end{tabular}

carried out with three different grids, whose streamwise and spanwise cell densities vary. The parameters are given in Table 4.1. The discrete test filter is implemented according to the trapezoidal rule and Simpson rule in the dynamic model. An instantaneous flow field of a very fine grid simulation is analyzed in order to study the discretization error present in the large-eddy simulations.

\subsection{Conclusions}

The channel flow at $R e_{b}=2800$ was computed first with no subgrid-scale (SGS) model by employing three different mesh sizes. The coarse-grid calculations overpredicted the peak of the turbulent energy and under-predicted the wall stress. Figure 4.2 shows that streamwise stresses are over-predicted and wall normal and spanwise stresses are under-predicted with inadequate resolution. Consequently, the wall stresses are under-predicted with the coarse grids. It is known from the experiments and the simulations, that in the spanwise direction there exist streaks of high and low-speed fluid with a mean spacing of 100 wall units [20]. It is probable that the coarse grid, with a spanwise node spacing of 36 wall units, is unable to capture these near-wall eddies correctly. With the fine grid, all the monitored quantities are quite close to those from the reference DNS.

Figure 4.2 shows the resolved normal stresses with the SGS models on the medium mesh. The eddy viscosity damps the flow and the wall stresses decrease further. In 


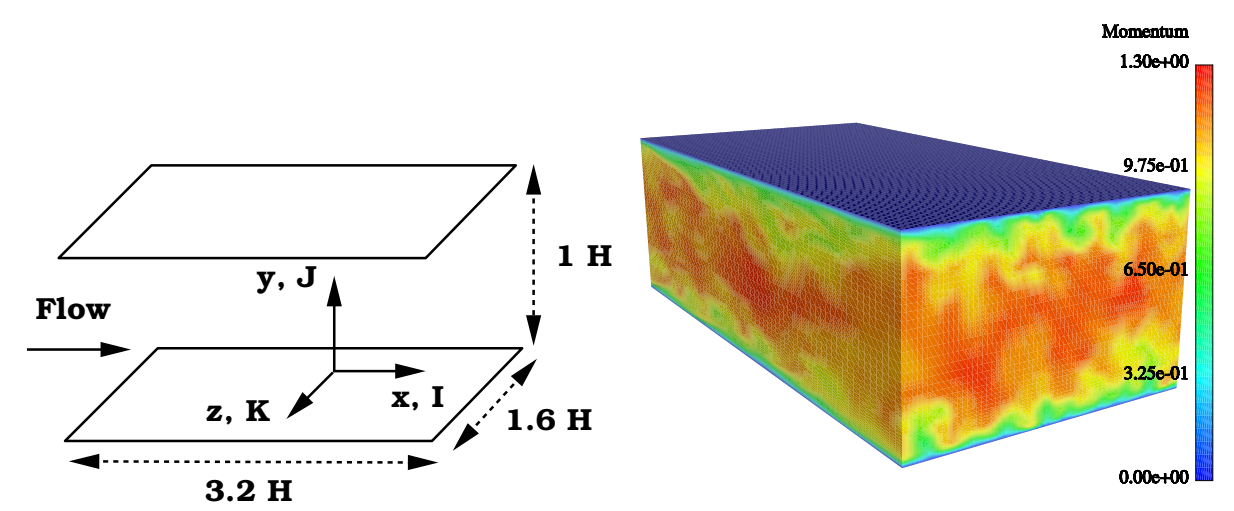

Figure 4.1: A scheme of the channel flow at $R e_{b}=2800$ on the left. An instantaneous picture of the flow coloured with momentum on the right.
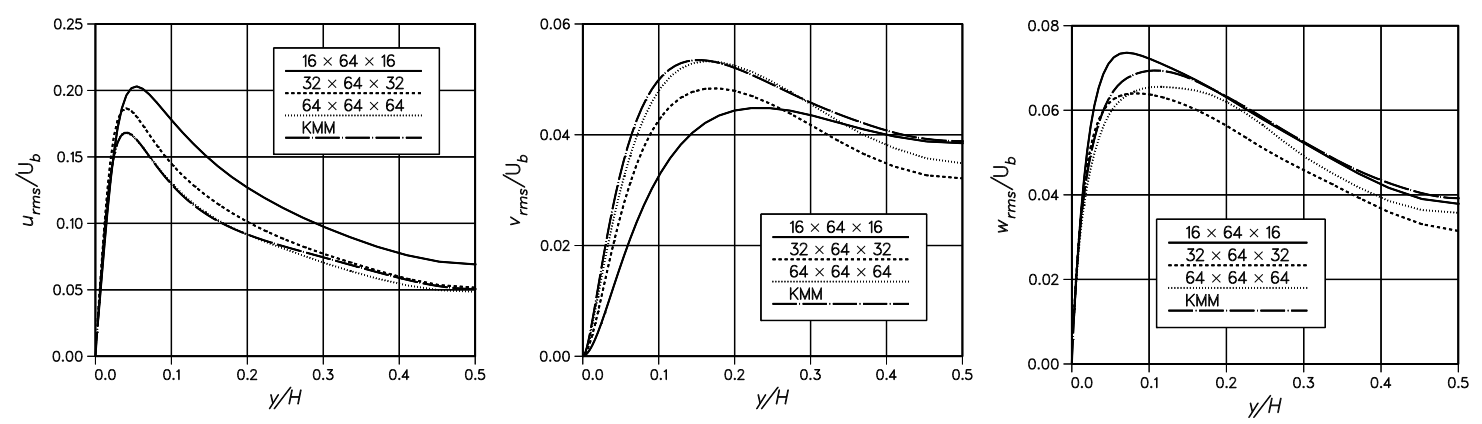

Figure 4.2: Resolved normal stresses from the non-modelled simulations with different grids at $R e=2800$. 
addition, the result from the fine grid is altered correspondingly. The eddy viscosity from all the SGS models is decreased with the decreasing mesh spacing, but apparently this 'laminarization' process is inadequate for all the SGS models applied. Najjar and Tafti [26] used a staggered mesh with a second-order Harlow-Welch scheme and a fifth-order upwind-biased scheme for the convection term in their study. They concluded that the dynamic model improved the prediction capability of their result, when used with the Harlow-Welch scheme and with a minimum resolution that would capture energy-producing mechanisms. This study, performed with a colocated mesh and a second-order central discretization, indicated no improvements at any grid level tested. Normalizing the results by the shear velocity is not proper, since it varies from one calculation to another. Ignoring this fact might lead to false conclusions, especially in assessing the influence of the SGS model on the resolved turbulent quantities. The filtering operation affects the model output; in the current dynamic models the top-hat filter is integrated with the three-point trapezoidal and Simpson quadrature. The former produces about $30 \%$ greater viscosity in the core flow than the latter.

At the higher Reynolds number of 12500 the channel flow was computed by utilizing one grid size only. The trend in the results was similar to that of the low Reynolds number case. The streamwise fluctuations are over-predicted and the friction is under-predicted. The non-modelled computation oscillates non-physically and the SGS models stabilize the computation and improve the results, especially in the core flow where the cell volumes of the stretched grid are large. The simple Smagorinsky model accompanied by the van Driest damping function works best among the applied models. In the dynamic model the numerical implementation has a great effect. The volume over which the eddy viscosity is calculated becomes relatively large. The resolution that has been applied to the case might be too coarse for the dynamic model. On the other hand, a second-order finite-difference approximation includes a lot of numerical truncation errors as small filtering molecules are applied. A longer filter cuts the subgrid frequencies more sharply but the proper implementation is more tedious and the resolution of the computation should probably 
be finer.

The discretization error was assessed at $R e_{b}=2800$. A procedure similar to that used by Vreman et al. [35], [36] was undertaken to estimate the discretization error and the subgrid term. The case at $R e_{b}=2800$ is computed with a dense grid size of $128 \times 64 \times 128$. The filtering operation to this field, which is assumed to an accurate DNS result, yields

$$
\frac{\partial \overline{u_{i} u_{j}}}{\partial x_{j}}=\frac{\partial \bar{u}_{i} \bar{u}_{j}}{\partial x_{j}}+\frac{\partial \tau_{i j}}{\partial x_{j}}
$$

where the SGS stresses are given by

$$
\tau_{i j}=\overline{u_{i} u_{j}}-\bar{u}_{i} \bar{u}_{j}
$$

The above derivatives are estimated with a fourth-order scheme in the homogeneous directions. In the wall-normal directions the derivatives are estimated with a secondorder scheme, which takes into account the grid stretching. In a coarse LES grid the discretization error is introduced also in the pressure term, whereas the diffusion term is ignored, since it is smaller by the magnitude of the Reynolds number. The relation of the discretized $i$-direction convective terms between two resolutions can be written as

$$
\frac{\partial \bar{u}_{i} \bar{u}_{j}}{\partial x_{j}}+\frac{1}{\rho} \frac{\partial \bar{p}}{\partial x_{i}}=\frac{\delta \bar{u}_{i} \bar{u}_{j}}{\delta x_{j}}+\frac{1}{\rho} \frac{\delta \bar{p}}{\delta x_{i}}+\varphi_{i}
$$

where $\delta / \delta x_{i}$ denotes for the second-order finite volume operator used in the simulations and $\varphi_{i}$ is the error between the fine and coarse level approximations.

An instantaneous flow field was filtered by a trapezoidal formula by using 6 and 10 cells in each homogeneous direction. The filters are then 5 and 9 grid-spacings wide, respectively. The filtered variables coincide at the wall-normal plane in the grid nodes of the dense grid, and at the cell centre nodes of the $32 \times 64 \times 32$ grid. At the coarse mesh level the filters are 1.25 and 2.25 grid spacings wide, respectively. In the wall normal direction the variables are not filtered. The accurate subgrid-scale stresses can be defined from Eq. (4.2). The discretization error $\varphi_{i}$ can be estimated from Eq. (4.3) in each co-ordinate direction. The $L_{2}$-norms of $\varphi_{i}$ and $\partial \tau_{i j} / \partial x_{j}$ are presented in Fig. 4.4. The quantities are computed over the $x z$-planes at one 
instant of solution. The estimated error dominates the accurate subgrid term by a factor of ten even if the filter width is twice that of the grid spacing. Only in the vicinity of the walls are both terms about equal in size. A perfect SGS model will not necessarily improve the result in this case. However, a dissipation caused by the eddy viscosity might stabilize the computation and remove unphysical oscillations, as it does at $R e_{b}=12500$.

Najjar and Tafti [26] concluded that an improved result must not be taken for granted by applying a subgrid scale model in a finite-difference calculation. If the resolution of the grid is fine enough to provide a physically reasonable solution without any dissipation, the present results support the same conclusion. Studies with higher Reynolds numbers are required to clarify the advantages of the SGS modelling in finite-volume solutions. 

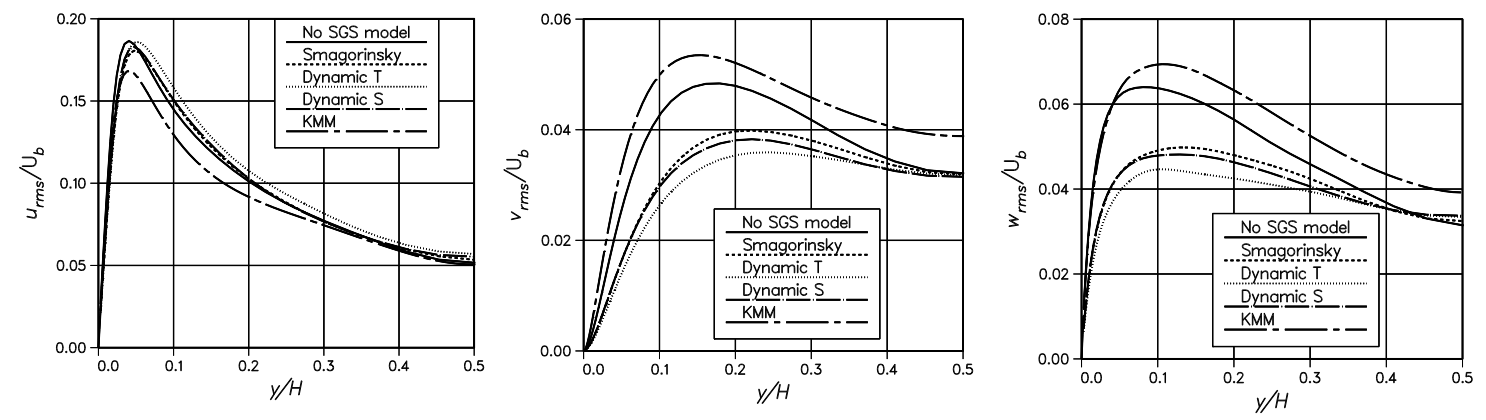

Figure 4.3: Resolved normal stresses from all the simulations with the medium grid at $R e=2800$.
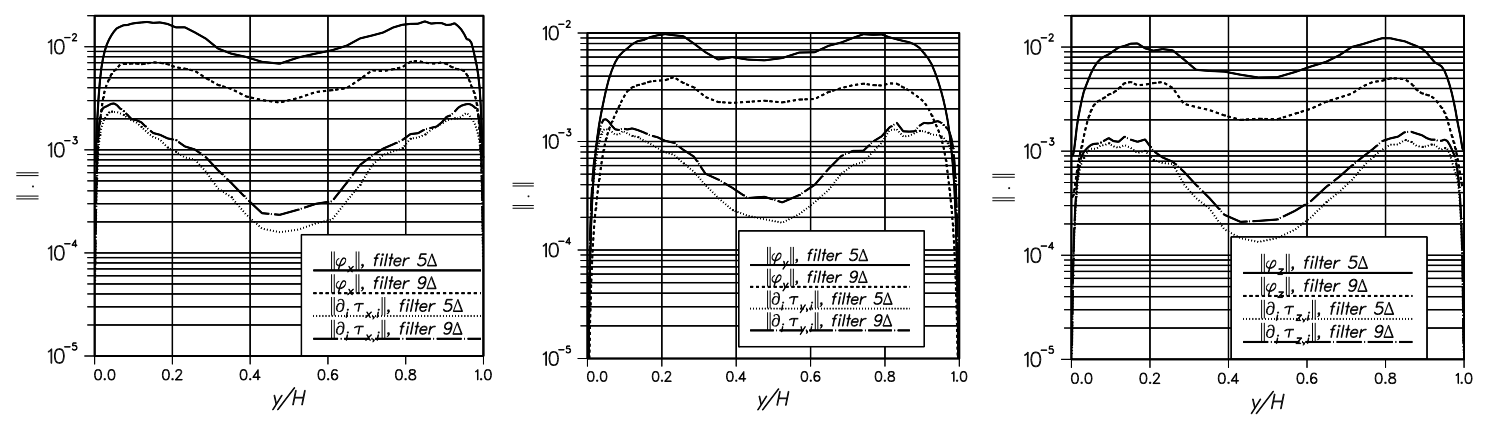

Figure 4.4: The $L_{2}$-norm of the estimated discretization error $\left\|\varphi_{x}\right\|$ and the subgrid term $\left\|\partial \tau_{x, x_{i}} / \partial x_{i}\right\|$ in the streamwise, wall-normal and spanwise direction, respectively. 


\section{Parallel Solver}

\subsection{Motivation and Background}

The channel flow described in the previous chapter is a good case for numerical and theoretical studies in turbulence. Most flows encountered around us are certainly more complex in terms of the flow behaviour and the domain. Furthermore, characteristic Reynolds numbers are often a lot higher than those seen in this thesis so far. In a parallel computer a computational task is divided between many processors. In an ideal case, the single-processor execution time is decreased to the time of the original time divided by the number of the processors used. In this section, a parallel multi-block solver for incompressible flow is described and validated. A spatial discretization is based on the co-located and structured finite-volume method with Rhie and Chow pressure coupling. The three-level implicit stepping is applied in time advancement. A computational domain is divided into separate blocks, which are assigned in separate processors. The Message-Passing Interface (MPI) is used for exchanging the boundary data. Several computations are performed to validate the solver in Paper III.

\subsection{Test Computations}

\subsubsection{Steady Cavity Flow}

The first test case is a steady-state cavity flow $R e=\frac{U_{w} L}{\nu}=400$. A first-order Euler method is used to iterate a converged result and a second-order central discretization scheme is applied for the convective and diffusive terms. The grid points are equally distributed and the number of cells is $96 \times 96 \times 8$, where the cells in the $k$-direction are set for computational reasons, specifically for the multigrid solver. The iteration is stopped until the $L_{2}$ norm of $u$-velocity was decreased below $10^{-10}$. With eight processors the number of iterations is increased by $10 \%$ compared to that needed by the single processor computation with a single domain. The CPU time is decreased more than linearly as the number of processors is increased. Figure 5.1 at the left 
shows that the domain decomposition does not deteriorate the solution. The superlinear speed-up shown at the right is probably due to the more efficient usage of a cache memory the processor is capable of with the smaller domain assigned to it.
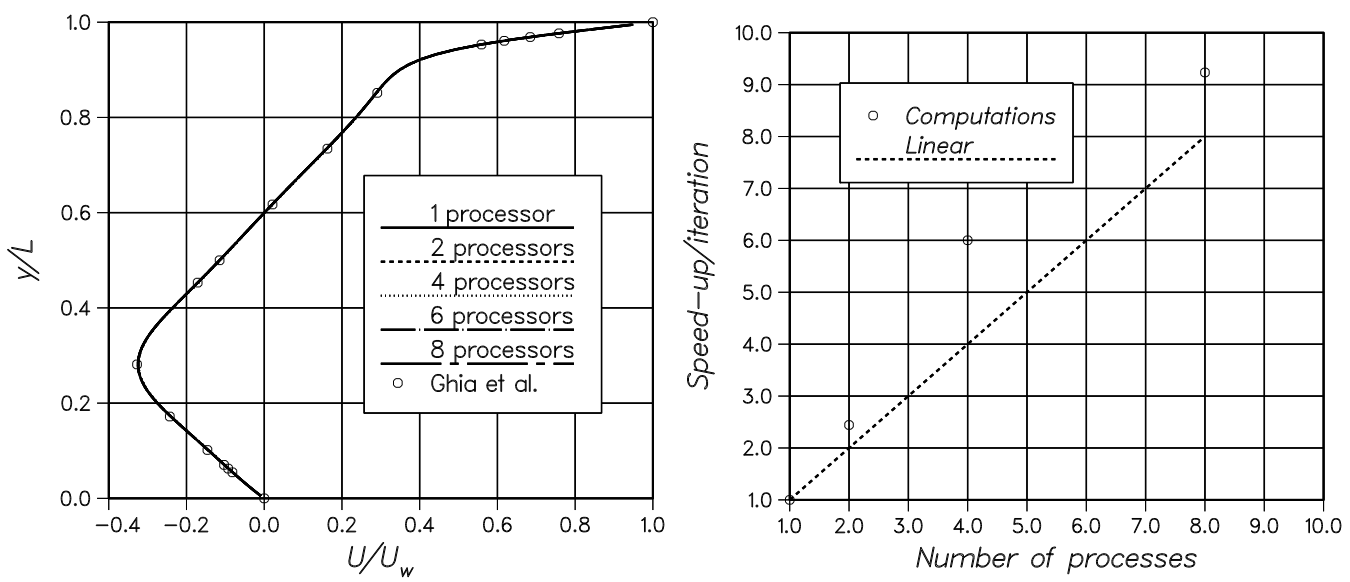

Figure 5.1: Cavity flow at $R e=400$. Converged $u / U_{w}$-velocity along the vertical centreline in the left and the speed-up per iteration. Reference result by Ghia et al. [11].

\subsubsection{Turbulent Cavity Flow}

The second test case is a cavity flow at a Reynolds number of 10000 . The width of the cavity $(W)$ is half of the length and the height $(L)$ of the cavity. This flow has been studied experimentally by Prasad and Koseff [29].

The grid contains $64 \times 64 \times 32$ grid cells and it is geometrically stretched from the wall. The domain is partitioned in equal 1, 2, 4, and 8 sub-domains which are assigned in separate processors. Large-eddy simulations were calculated with and without a subgrid-scale model. The $L_{1}$-norm of the mass balance is iterated to be smaller than $10^{-8}$. With four processors a non-modelled computation requires 9.6 iterations and a modelled one 5.8 iterations per time step for this criterion. Fig. 5.2 represents the results along the vertical and horizontal centreline. The computations over-predict the thickness and the maximum velocity on the downstream and especially on the upstream wall. The insufficient resolution of the interior coarse 
grid might cause the discrepancies between the computed and the measured data. The dynamic model does not affect the results much on the vertical and horizontal centerlines. The model computes the largest viscosity near the downstream eddy, where the flow is turbulent. The speed-up is again superlinear although not as strongly as in the steady-state case where the mesh is smaller.
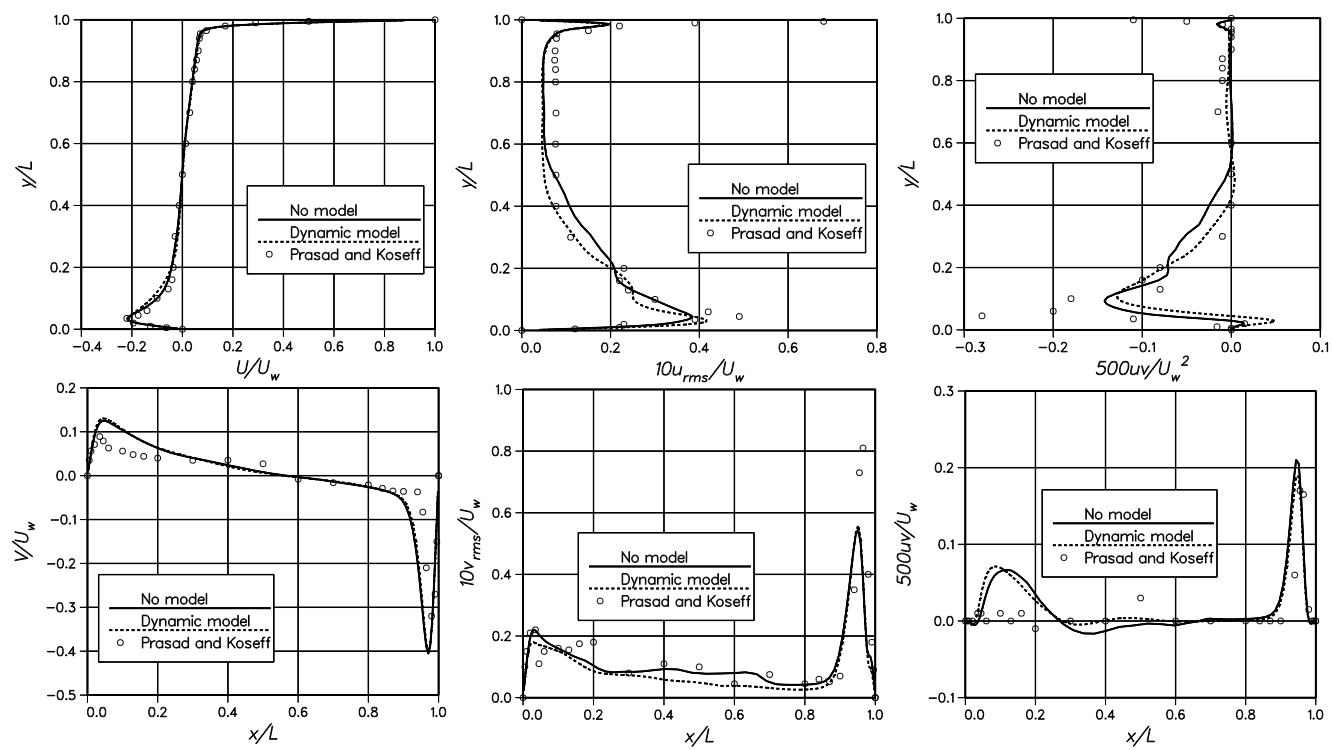

Figure 5.2: Cavity flow at $R e=10000$. Top : $U / U_{w}$-velocity, $10 u_{r m s} / U_{w}$ and $500 u v / U_{w}^{2}$ along the vertical centreline. Bottom : $V / U_{w}$-velocity, $10 v_{r m s} / U_{w}$ and $500 u v / U_{w}^{2}$ along the horizontal centreline.

\subsubsection{Fully Turbulent Pipe Flow}

The third test case is a fully turbulent pipe flow at a friction velocity based Reynolds number $R e_{\tau}=\frac{u_{\tau} D}{\nu}=621.4$, which was set by forcing the flow with a constant body force. This approximately equals $R e_{b}=\frac{U_{b} D}{\nu} \approx 10000$, where $U_{b}$ is the bulk flow velocity and $D$ is the diameter of the pipe. The shortest mesh is $1.2 \mathrm{D}$ long and consists of 5 blocks, each containing $32 \times 32 \times 32$ cells, altogether 163840 cells. The height of the two cells next to the wall is approximated to be around one in wall units $\left(r^{+}=1\right)$ from the law of the wall. The stretching ratio varies between 
1.075-1.087 in the four outer blocks. The azimuthal mesh spacing $R \Delta \theta^{+}$at the wall is 15 and the streamwise mesh-spacing $\Delta z^{+}$is 24 . This flow was first computed by using one Power4 processor, after which all blocks were assigned to separate processors. The basic mesh was then multiplied and connected together, so that the computations were performed by using multiple pipe lengths up to 30 processors. The pipe flow may be considered a simple flow physically, but computationally it possesses many complex features for the current Cartesian solver. Non-modelled large-eddy simulations were calculated first. Within the time step of $0.05 T$ ( $T=$ $\left.D / U_{b}\right)$ eight subiterations were taken, which was considered to provide a sufficient convergence. The period during which the statistics were gathered varied between $200 T$ and $500 T$ depending on the mesh size. In the end, the data was averaged also in the axial and radial directions. The velocity profile is slightly flatter than the measured one. The streamwise rms fluctuations are over-predicted whereas the radial $\mathrm{rms}$ fluctuations are under-predicted, as seen in Fig. 5.3. The fluctuations obtained from the simulation using the shortest pipe length $1.2 \mathrm{D}$ deviate somewhat from the rest of the results. A localized SGS model was utilized with the pipe
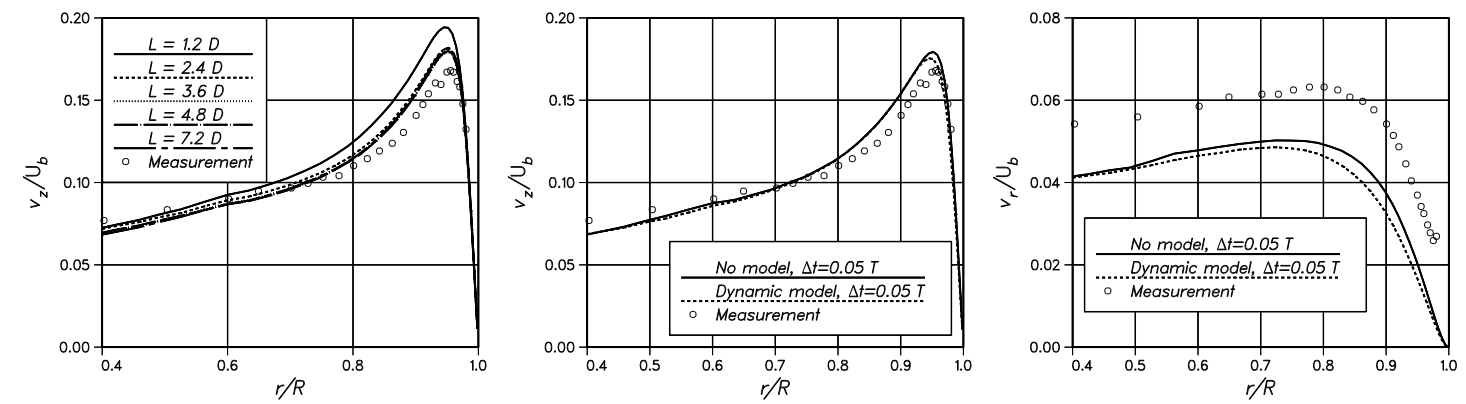

Figure 5.3: Results from the pipe flow at $R e_{\tau}=621.4$. At left streamwise fluctuations with different mesh lengths. The effect of the SGS model on the streamwise and radial fluctuations at the centre and the right, respectively. The reference measurements by den Toonder and Nieuwstadt [9].

length of 3.6D. The model dampens slightly the resolved turbulent intensities as shown in Fig. 5.3. Near the wall the model dampens itself excluding the need for the 


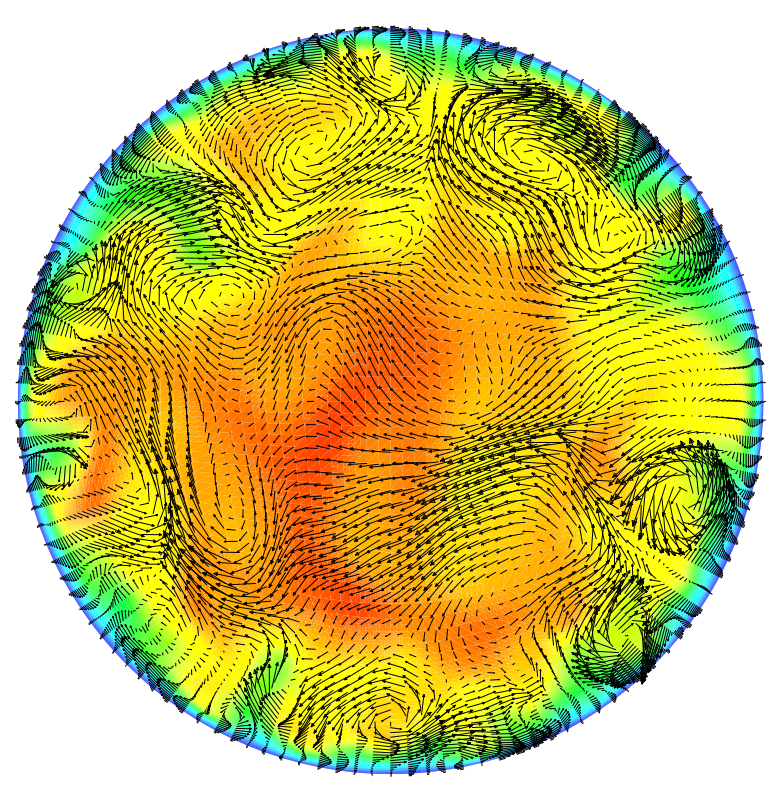

Figure 5.4: Instantaneous momentum vectors projected to a cross-section in a pipe flow at $R e_{\tau}=621.4$.

van Driest damping function, which requires a distance from the wall. In complex geometries the definition of the wall distance may be ambiguous. The scale-up of the parallelization was linear in these calculations.

\subsubsection{Conclusions}

A parallel, finite-volume solver was developed for large-eddy simulation. The algorithm is based on a traditional SIMPLE technique and an implicit iterative time stepping is applied. The implicit part of the solution is solved independently in each block and process and the boundary values are exchanged only after each outer iteration loop. This simplifies the code a lot without much affecting the convergence. Also, the amount of the message passing between the processes is reduced. The parallelization of the present solver shows good scaling in the present ideally balanced computations. Several flows are simulated to test the solver. The local dynamic model reduces the viscosity correctly in the vicinity of the wall without any ad hoc damping functions. 


\section{Jet in a Cross-Flow}

\subsection{Flow Configuration}

The jet in a cross-flow considered here has been experimentally studied by Crabb et al. [6]. The Reynolds number $R e_{D}=46700$ referred to the pipe flow is rather large for an LES of the whole jet. Therefore, the computational domain has been reduced from that of the wind tunnel used by Crabb et al.. The jet-to-cross-flow velocity ratio $J=2.3$. The setup of the jet in a cross-flow is sketched in Fig. 6.1. The number of the control volumes $(\mathrm{CVs})$ is $192 \times 96 \times 144$ in the stream-wise, wall-normal and span-wise directions, respectively. In addition to this, a jet pipe is represented with $110592 \mathrm{CVs}$, altogether $2764800 \mathrm{CVs}$, which are equally distributed into 25 blocks. The grid is clustered around the jet exit and the height of the first cell from the lower wall is $0.002 D$, which corresponds to $\Delta y^{+}=4.7$ if scaled by the friction velocity of the incoming turbulent pipe flow. The stretching factor in the wall-normal direction is less than 1.06. Crabb et al. found that without the jet, the height of the

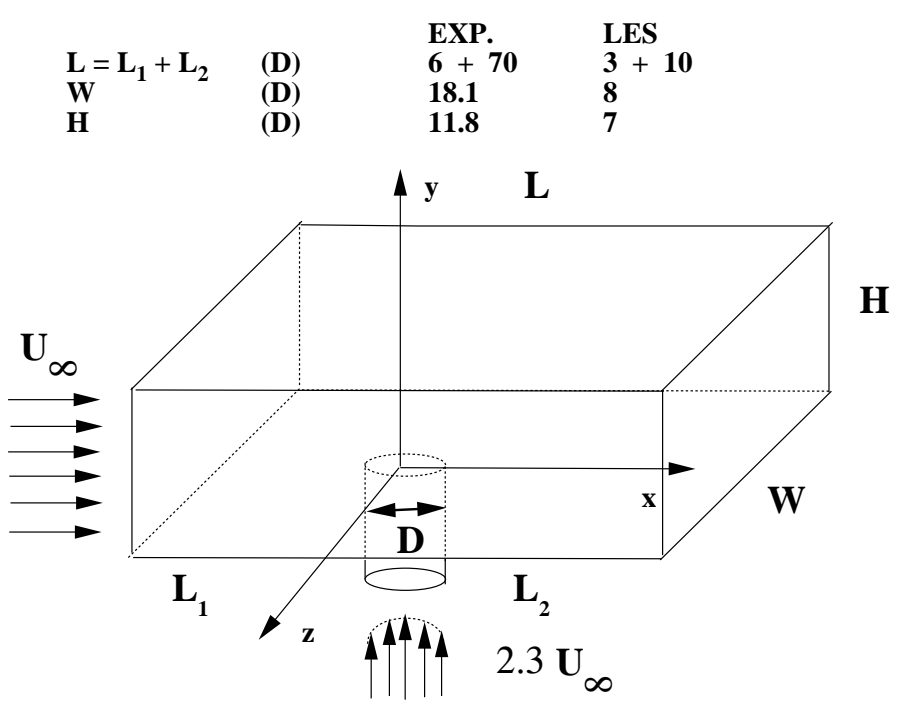

Figure 6.1: Schematics of the domain in the jet in a cross-flow. The experiment refers to the wind tunnel used by Crabb et al. [6] whereas the LES refers to the present computational domain size. 
boundary layer in front of the jet exit was about $0.24 D$ high and the free-stream turbulence level of the channel is $0.6 \%$. Two different boundary conditions are applied. First a uniform cross velocity $U_{\infty}$ was set at the inlet.

An unsteady condition was extracted from a turbulent profile from half the channel flow $0.22 D$ high. Above this boundary layer, a non-shear turbulent flow was extracted from the middle of the channel flow so that the intensity was gradually damped to zero by $y / D=0.5$. Above that, a constant value was used preserving the same mass flow through the inlet as the steady-state boundary condition. At the lateral and top surfaces, free-slip boundary conditions were applied and a no-slip condition was forced at the bottom wall. At the outlet a zero-gradient (Neumann) condition was used. A zero-gradient pressure surrounded the whole area and the average pressure level in the area was fixed.

First the case was calculated with a steady fully developed turbulent profile. The unsteady condition was obtained from an LES of a fully developed pipe flow. The topology of the mesh and the time-step size were different in the precursor computation. Therefore, the boundary condition velocities were interpolated spatially and temporally in the jet computation. The sequence of the boundary values was also interpolated to be periodic, whose duration corresponds to the bulk flow advancement of two diameters in the inlet pipe.

Crabb et al. seeded the jet with helium trace and measured the mixture fraction $\theta / \theta_{J}$ at different locations downstream. In the cross-flow inlet $\theta / \theta_{J}=0$ and in the jet $\theta / \theta_{J}=1$. A zero-gradient for the scalar was set at all the other boundaries. The turbulent Prandtl or Schmidt number was set to a constant value of 0.6, which is in the range suggested in [5].

The startup of the calculation was quite difficult. The dynamic model did not stabilize the computation and consequently it was not used. An excessive value for the Smagorinsky constant $C_{s}$ was set, until after some transient time, it was lowered to a value of 0.17 . Also, an under-relaxation of a few per cent was added to the diagonal of the pressure correction equation in order for the MG solver to converge. The time step was rather short, $\Delta t=0.005 T$, where $T=D / 2 U_{b}$. This implies that 
the bulk cross-flow advances from the inlet to the outlet during 5200 time steps. Due to the relatively thin cells, the highest $C F L$ values are around 4 at the jet exit, however. A total of 15 sub-iterations was calculated during a time step. It might have been possible to obtain the same results with a smaller number of iterations, but this was not tested. The global mass balance residual reduced to a third from the first iteration, which is a rather poor convergence within a time step. Since the time step is globally so small, a global mass error remains small even at the first iteration cycle. The flow was computed for a time of $113 \mathrm{~T}$ from the initial state before statistics were gathered. The statistics were gathered during $153 \mathrm{~T}$, or six flow-through times. The results were relatively converged after $87 T$, especially near the jet exit. The biggest changes were seen in non-diagonal stresses in the far field at $x / D=8$. There was no need to reconsider the conclusions due to the continued computation, however. In calendar time all this corresponds to approximately 40 days when using 25 Power4 processors of an IBM SP cluster. With the unsteady boundary condition the statistics were gathered a time of 7.5 flow-through times.

\subsection{Results}

\subsubsection{Flow Field}

In this section, a qualitative overview of the flow field is given. The mean flow streamlines in the jet exit region are shown in Fig. 6.2. There are some differences between the solutions with the steady and the unsteady BC at the inlet pipe, which from now on are referred to as LESSBC and LESUBC, respectively. Both cases are referred to as LES in general. In the central plane upstream of the jet exit there is a single vortex $\left(V_{1}\right)$, whose centre is located $0.25 \mathrm{D}$ upstream of the lip. Kelso et al. found that at a smaller Reynolds number $U_{\infty} D / \nu=1600$ there were two vortices in front of the jet, separated by a saddle point. They also visualized a 'hovering vortex' above the jet exit. The vortex originates from the collision of the jet and the cross-flow shear layers. In Fig. 6.2 the streamlines of the LESSBC reveal three distinguishable roll-up vortices $\left(V_{2}, V_{3}, V_{4}\right)$, two of them inside the pipe $\left(V_{3}, V_{4}\right)$. In 
LESUBC the roll-up vortex $V_{2}$ is not present and $V_{4}$ is hardly distinguishable. In both schemes some part of the cross-flow near the surface is swept into the pipe. The saddle point $S$, or the lowest point where the incoming fluid penetrates, is located at approximately $y / D=-0.2$. The LESUBC shows a smaller separation bubble inside the inlet pipe. At a Reynolds number ten times smaller, and at the jet-to-cross-flow velocity ratios $R=2.2$ and 4.0, Kelso et al. [18] reported the saddle point locations of $y / D=-0.4$ and -0.16 , respectively. At high velocity ratios $(\geq 6)$ the saddle point is located at the lip. In the symmetry plane downstream of the jet, there resides a node $(N)$. LESUBC predicts the location of the node slightly higher $(x / D=1.1, y / D=0.21)$ than $\operatorname{LESSBC}(x / D=1.06, y / D=0.18)$. A vortex is also located right downstream of the jet exit edge $\left(V_{5}\right)$. Behind the jet there is a rather strong back-flow near the flat plate. The back-flow almost reaches a velocity of the free-stream value. The cross-flow deflects over the bending jet and accelerates to a value of nearly twice the free-stream value. The mean vertical velocity possesses two regions of strong upward motion. The upper region is generated directly by the jet. In the lower region the upward velocity is at maximum about half of the jet velocity. This motion is the fluid flowing from the node toward the jet trajectory. Yuan et al. [40] found that at $R e_{D}=2100$ there was a clear distinction between the sign of the vertical vorticity emerging from the left-hand side and the right-hand side of the pipe [40]. This notion is confirmed in this case. The vorticity seems to mix quicker in the present case, which is probably due to the higher Reynolds number. Figure 6.3 reveals the vortices with the iso-surface of the second invariant of the velocity gradient $Q$ defined as

$$
Q=-\frac{1}{2}\left(\bar{S}_{i j} \bar{S}_{i j}-\bar{\Omega}_{i j} \bar{\Omega}_{i j}\right)=-\frac{1}{2} \frac{\partial \bar{u}_{i}}{\partial x_{j}} \frac{\partial \bar{u}_{j}}{\partial x_{i}}
$$

An animation shows that the vortex upstream of the jet exit is formed and it moves to the lip of the jet exit where it seems to merge with the vortices of opposite sign rising from the pipe. The merger may explain that at a distance of approximately one diameter above the exit there is weaker coherent vorticity in front of the jet. The animation also shows how shear layer vortex layers are shed one after another 


\section{LESSBC}

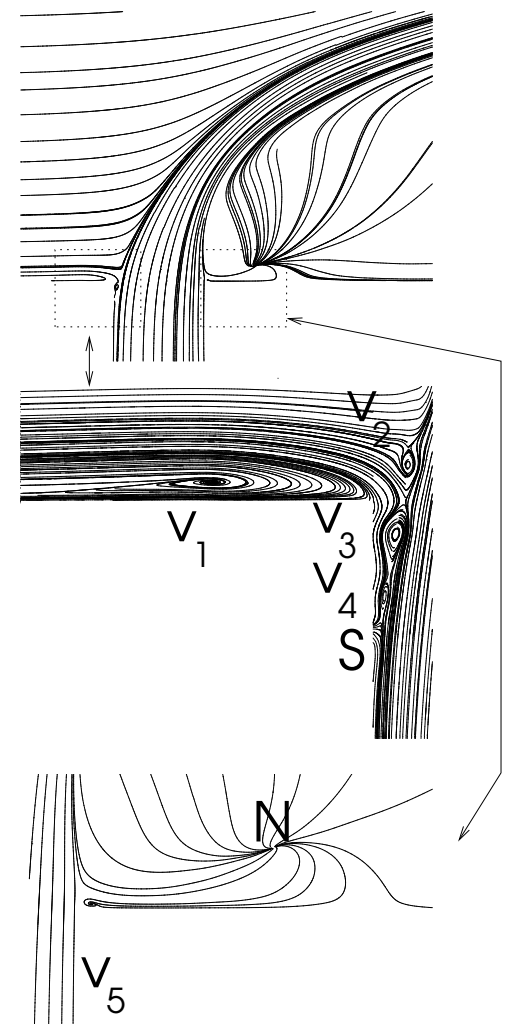

LESUBC

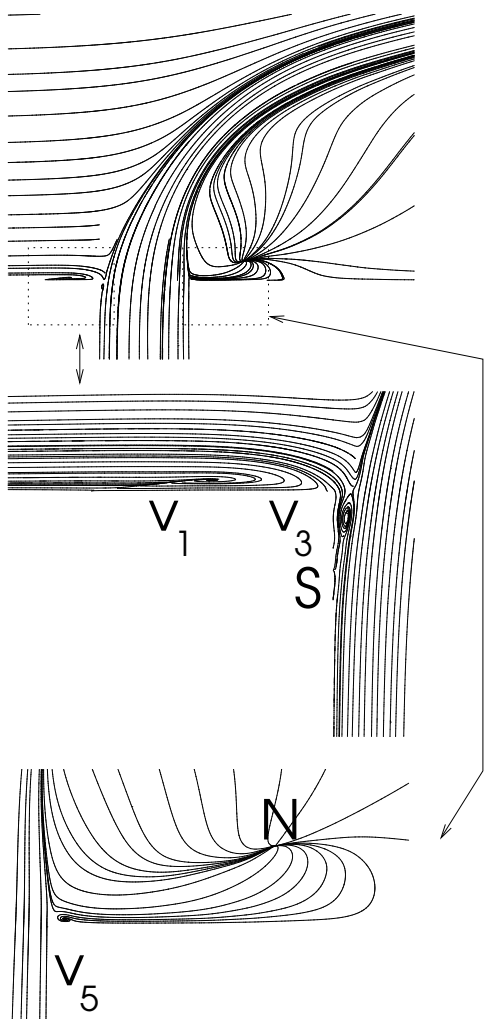

Figure 6.2: The streamlines of the mean velocities in the symmetry plane with different boundary conditions. In the second row magnifications upstream from the jet exit. The separation zone is smaller inside the pipe with LESUBC including only one vortex $V_{3}$. The lee of the jet (bottom) shows a node $(N)$ and a vortex $\left(V_{5}\right)$.

near the exit. They move along the jet before they lose their coherence at a distance of a few pipe diameters away from the exit.

Figure 6.4 shows an evolution of a counter-rotating vortex pair (CVP) at various distances from the jet exit. A small CVP is present already at the jet exit $(x / D=$ 0 ), which supports the idea that it is initiated by the pipe vorticity (see e.g. [18]). Further downstream the CVP grows and the computational domain may constrain the spreading slightly.

The resolved turbulent kinetic energy (TKE) in the symmetry plane (Fig. 6.5) 


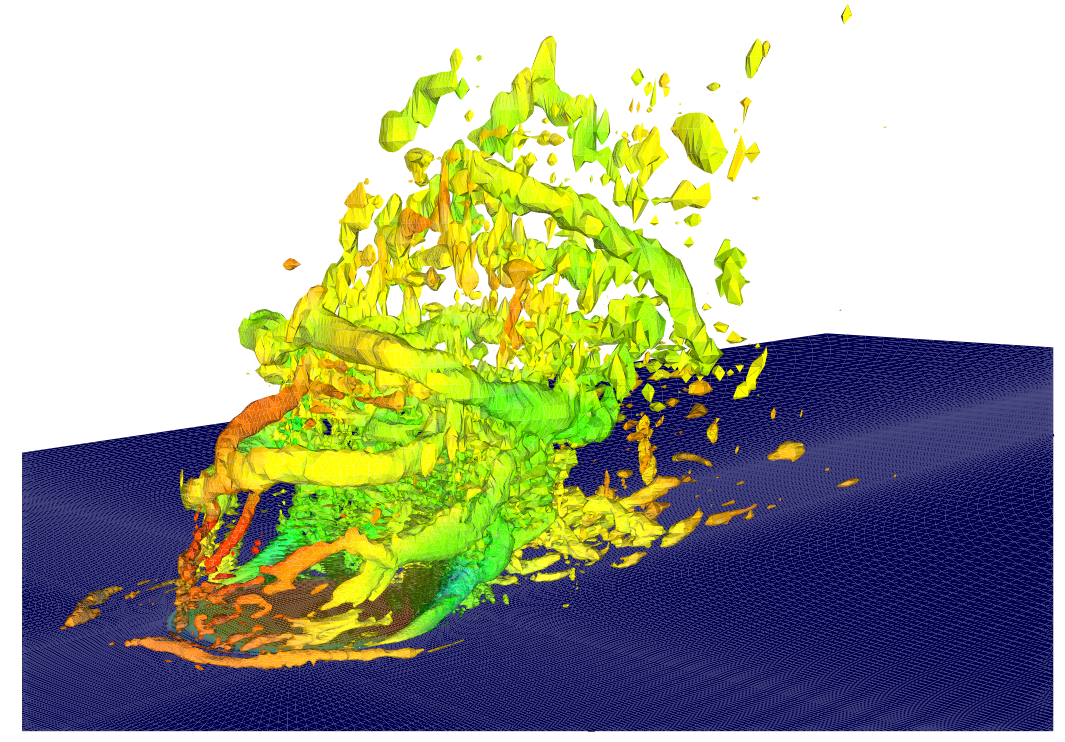

Figure 6.3: An instantaneous iso-surface of the second invariant of the velocity gradient $Q=30$.

shows a maximum under the jet trajectory where the flow emerging from the node curves to join the jet. Another smaller maximum is located at the node behind the jet. The eddy viscosity (Fig. 6.5) exhibits a local maximum under the jet trajectory too, approximately in the same location as TKE. At the node there is no maximum. This is prevented by a shorter length scale due to the grid clustering or a smaller strain rate, or both. The greatest viscosity ratio, approximately 35, exists in the shear layer near the inlet of the pipe. The resolution is far too coarse as the resolved TKE is almost zero in the boundary layer of the inlet pipe. Poor accuracy is obtained inside the pipe but we assume that this is not important in the simulation of the whole jet, which is the primary focus of the present work.

\subsubsection{Comparison to the Measurements}

A comparison of the calculated flow field to the experiment of Crabb et al. [6] (CDW) is conducted next. The mean stream-wise velocities in the central plane are shown in Fig. 6.6. At $x / D=-1$ the present LES shows a slight acceleration near the 


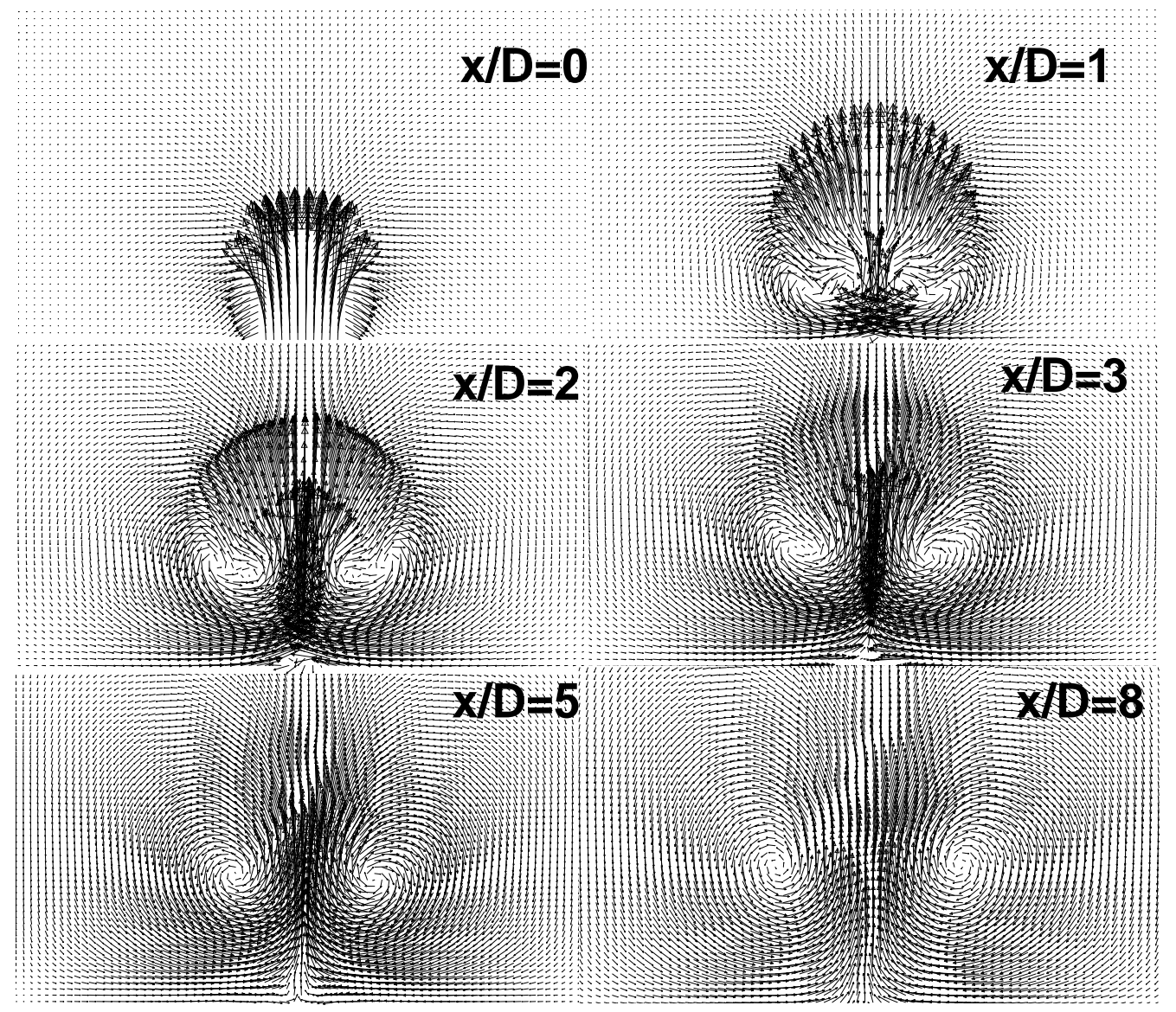

Figure 6.4: Evolution of the counter-rotating vortex pair at stream-wise planes at various distances from the jet exit.

flat wall at $y / D=0.1-0.2$, which is due to the vortex blocking the flow. See the close-up at bottom right in Fig. 6.6. The turbulent boundary profile of LESUBC results in a smaller peak and CDW shows no such effect. In a real flow, such a recirculation area may be closer to the wall. At the down-stream pipe wall $x / D$ $=0.5$ the LES predicts a small back-flow at $y / D=0.2$ (close-up). The positive velocity over the wall $y / D=0.02$ is connected to vortex $V_{5}$ described above. There are two minima seen in the back-flow at $x / D=0.75$ and 1 . In the LESUBC the back-flow is slightly increased. The strong back-flow near the wall nodes might be a sign of a non-physical phenomenon owing to too coarse cells for the present wall- 

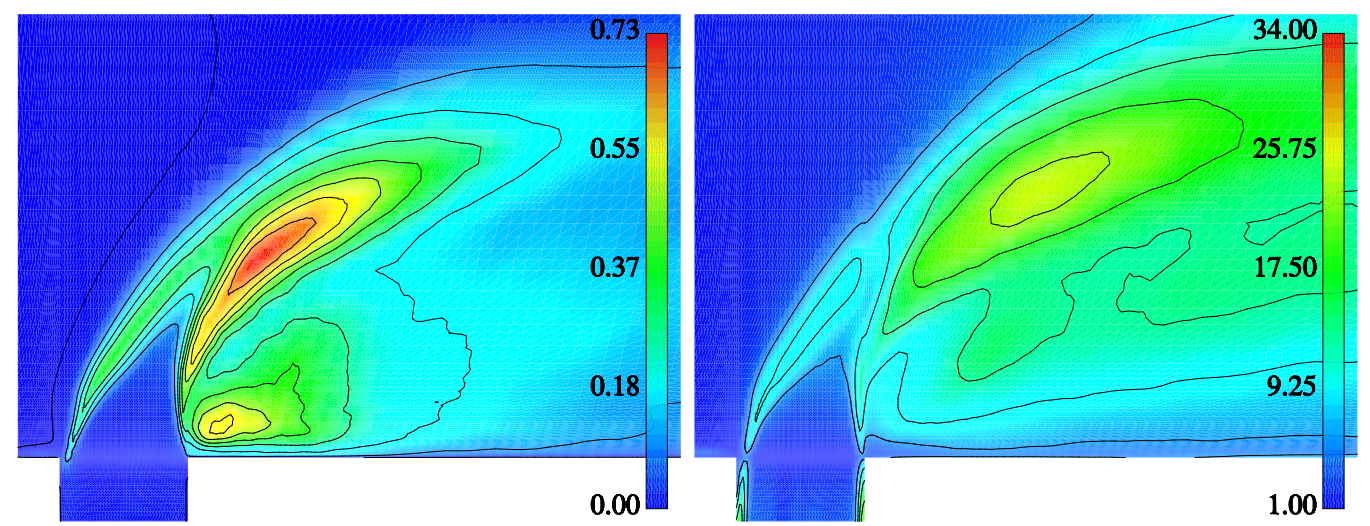

Figure 6.5: At the left turbulent kinetic energy $\frac{\overline{u_{i}^{\prime} u_{i}^{\prime}}}{2 U_{\infty}^{2}}$. At the right eddy viscosity $\frac{\overline{\nu+\nu_{s g s}}}{\nu}$ contours in the symmetry plane.

resolved LES. On the other hand, Andreopoulos and Rodi [1] mention that, in the lee of the jet with similar parameters, a reverse-flow region forms very close to the wall in which measurements were not possible. It is possible such a recirculation exists, but in any case the LES predict it too high up above the wall and probably too intense. In CDW, the back-flow resides approximately between $x / D=0.75$ and 3. In the LES there is no back-flow downstream of $x / D=2$ and the flow profile is flatter than that of CDW. The LESUBC shows a steeper gradient in the lee of the jet $x / D>3$.

The stream-wise turbulent intensity (Fig 6.7) upstream of the jet is somewhat difficult to interpret. Without the jet, the boundary layer width is $0.24 \mathrm{D}$ and the free-stream turbulence is less than $0.6 \%$. It is then questionable whether the measured turbulence level of $4 \%$ originates from the upstream boundary layer at $x / D=-1$. The fluctuations above the boundary layer width probably originate from the vortex motion described above or some other interaction of the jet and cross-flow. The LES captures only part of this intensity, which may be due to the applied Smagorinsky model.

The LESUBC has a peak value $u_{r m s} / U_{\infty}=0.05$ and LESSBC that of 0.02 near 

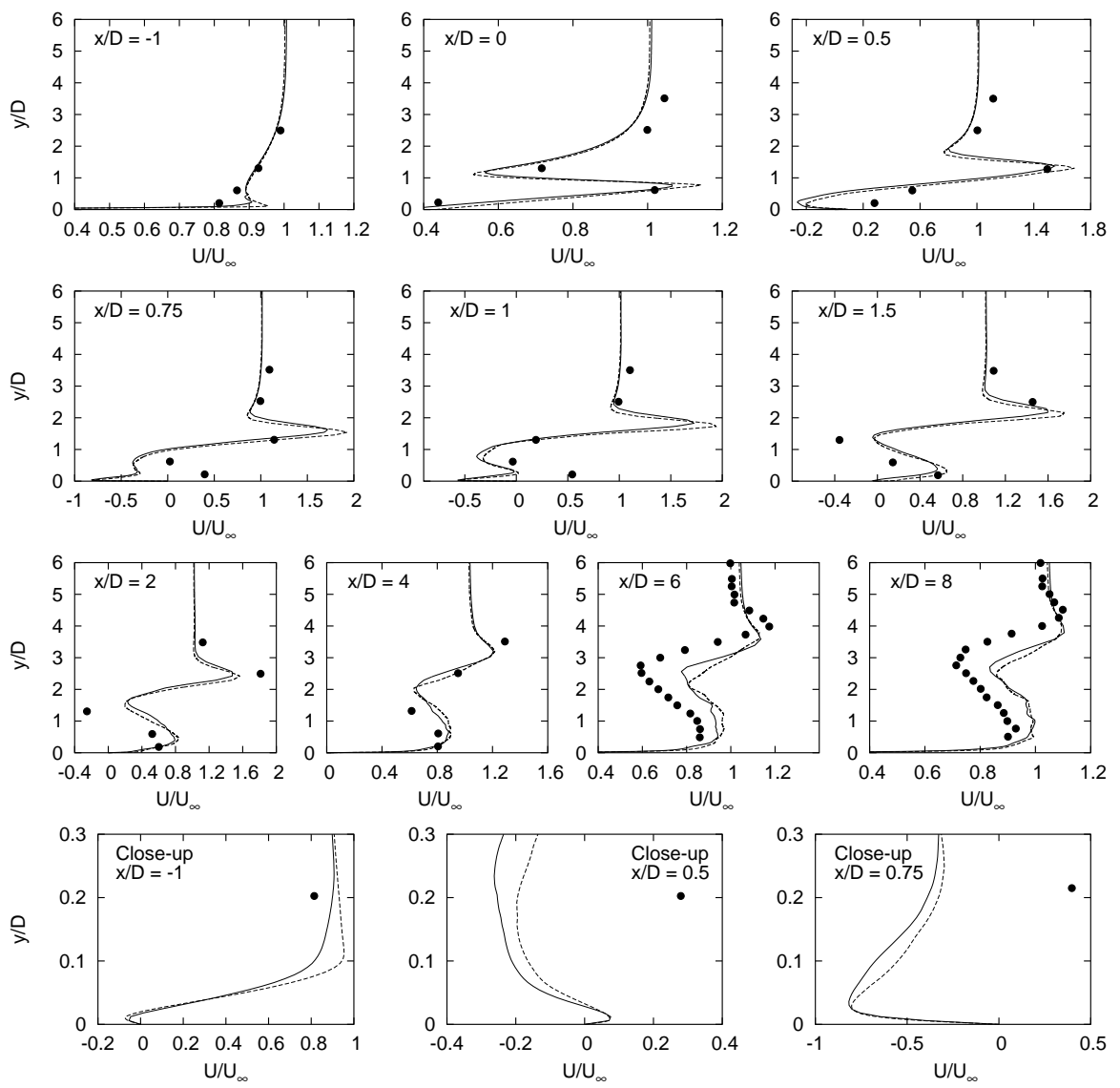

Figure 6.6: Mean stream-wise velocities in the central plane, $z / D=0$ : LESUBC $(-), \operatorname{LESSBC}(---), \operatorname{CDW}[6](\bullet)$.

the wall at $y / D=0.1$. Apparently, the large cells at the inlet due to the clustered mesh damp the fluctuations before interacting with the jet. The essential difference between the two cases at the cross-flow inlet is the average profile.

Downstream of the inlet there are two maxima in the intensity profile. They reside approximately at the location of the steepest gradient of the velocity. In general, the turbulent intensities are rather well predicted compared to the mean velocity.

Yuan et al. [40] performed simulations to test the effect of different inlet conditions. They tested a plug flow profile, a mean turbulent profile and a temporally evolving pipe flow and reported that the latter boundary condition obviously improved the result. However, they do not provide much evidence to support this conclusion. 

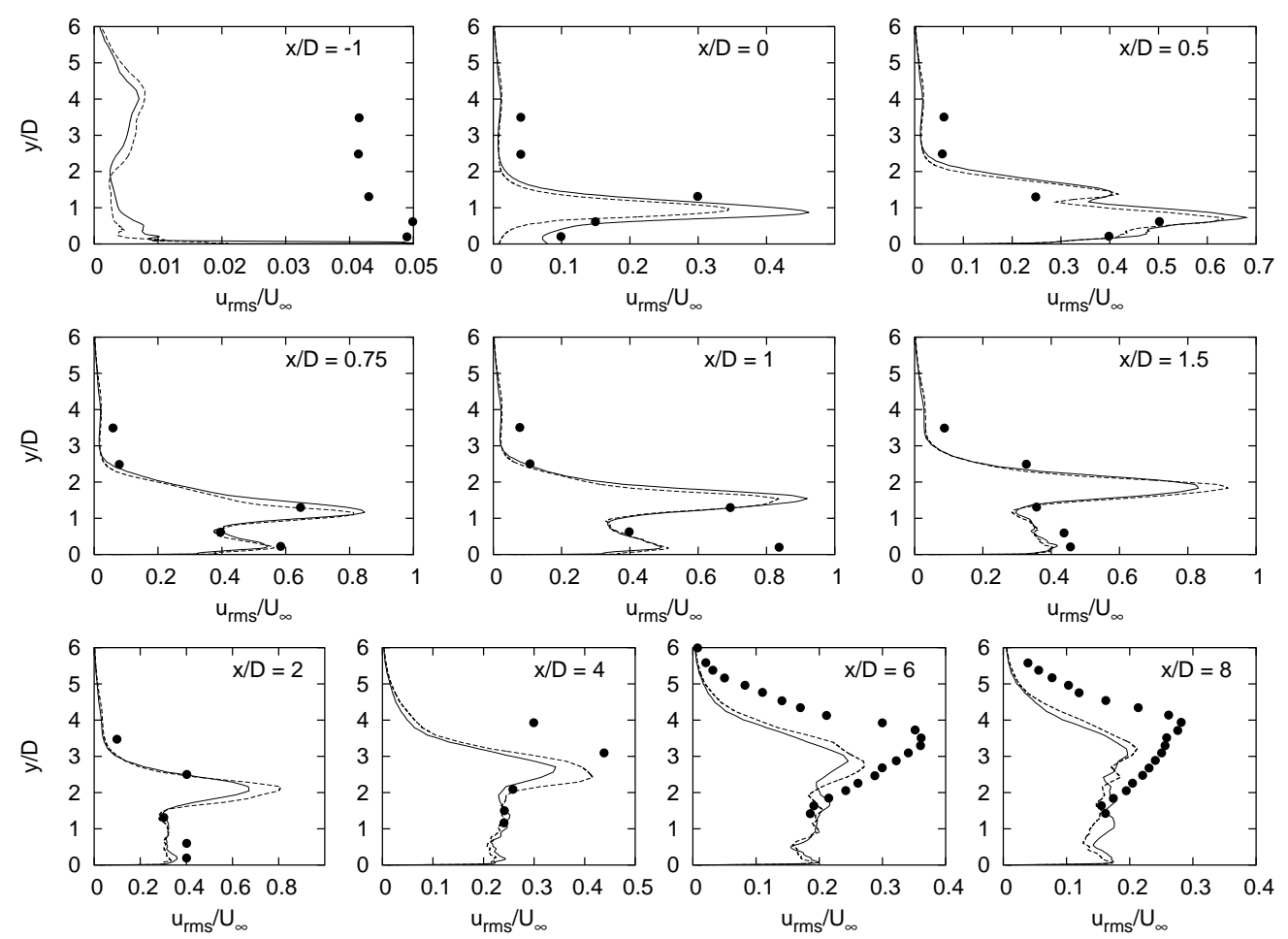

Figure 6.7: Stream-wise turbulence intensities in the central plane, $z / D=0$ : $\operatorname{LESUBC}(-), \operatorname{LESSBC}(---), \operatorname{CDW}[6](\bullet)$.

Wille [39] set the boundary condition at the wall by setting the lateral components to zero and the vertical component to such a value that the mean dynamic pressure was constant. Random fluctuations were added to the profile. This condition produced a surprisingly good agreement with CDW at $y / D=0.25$ although it prevented any upstream effect from the pipe. Fig. 6.8 shows the present jet profiles that have approximately $10 \%$ higher peak values than the measured one at $y / D=0.25$ and $z / D=0$. Crabb et al. [6] report that the profile at the outlet is a fully developed profile in a pipe whose length was $30 \mathrm{D}$. In the present simulation there are no significant differences in the outlet profiles between LESSBC and LESUBC. The LES shows a high velocity peak behind the jet indicating the intense recirculation. Half the diameter off the symmetry plane the LESUBC profiles are higher.

The difference between the two cases is apparent in turbulent intensities near the 


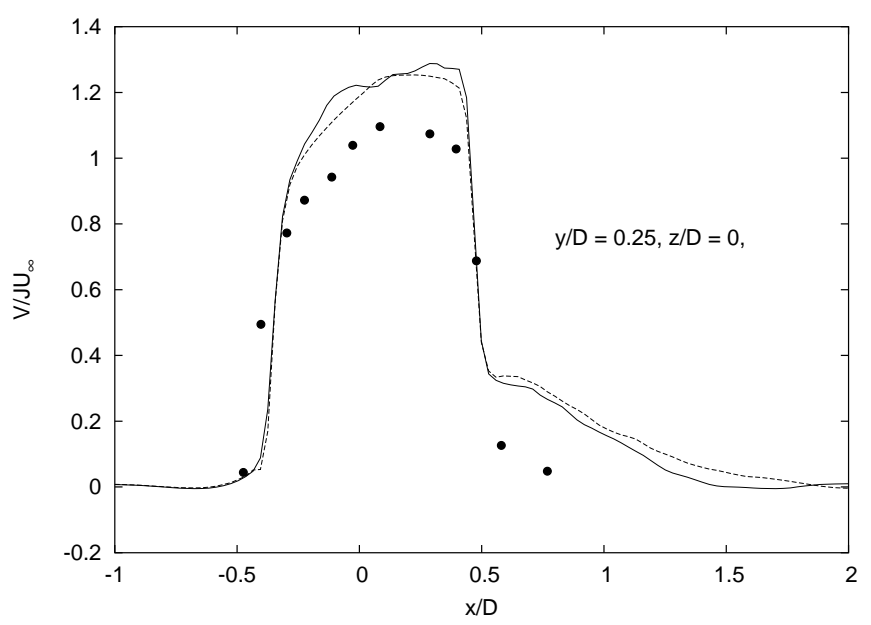

Figure 6.8: Jet profiles near the inlet at $y / D=0.25$ in the central plane, $z / D=$ 0 : $\operatorname{LESUBC}(-), \operatorname{LESSBC}(---), \operatorname{CDW}[6](\bullet)$.

jet exit in Figs. 6.9 and 6.10. The wall-normal intensity in Fig. 6.10 shows two distinct peaks near the jet exit, corresponding to the upstream and downstream shear layers of the pipe flow. Close to the wall $(y / D=0.25)$ the LESSBC predicts a very small intensity on the upstream side, whereas both the peaks are captured by the LESUBC. In the lee of the jet, the intensities are in a reasonable agreement with CDW. The back-flow generates fluctuations close to the wall also in the LESSBC, and on the upstream side the fluctuations grow closer to the measured ones further up, which is probably connected to the development of the ring vortices. In Figs. 6.9 - 6.10, only a resolved part of the turbulent stress is plotted. Also the total stresses including the contributions from the sub-grid scale model were gathered. Along the lines shown it is hard to distinguish the two quantities from each other as they lie within the line width. Wille [39] observed differences in the resolved and the total stresses with a coarse grid (88 440 cells) but the differences are reported to be very small with the fine mesh (997 920 cells). In the major part of the domain the modelled stresses are negligible compared to the averaged resolved ones. However, the resolved stresses consist of the large-scale motion, and the modelled stress is significant in stabilizing the calculation. In the shear layers (e.g. in the inlet pipe) 
the modelled stresses might be large even if compared to the resolved stress. The highest ratio $\nu_{s g s} / \nu$ of approximately 35 is calculated near the pipe wall where there is little or no resolved turbulence, as shown by Fig. 6.5. Here the near-wall grid is too coarse and the representation of the wall friction is inadequate.
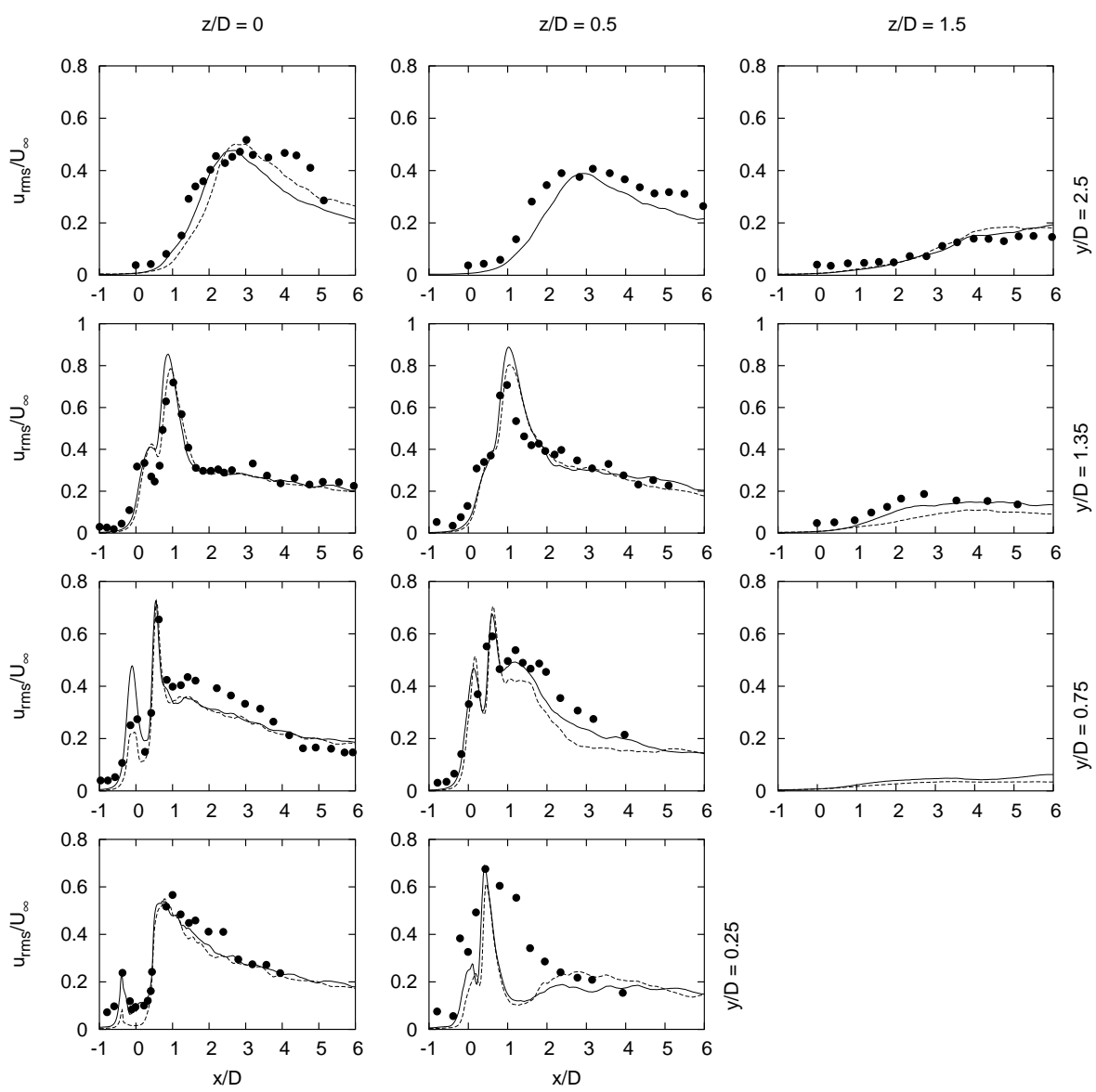

Figure 6.9: Stream-wise turbulence intensities plotted at various distances $y / D$ from the wall and $z / D$ from the central plane: $\operatorname{LESUBC}(-), \operatorname{LESSBC}(--)^{-}$, CDW $[6](\bullet)$.

Figure 6.11 presents lateral profiles for the plane $x / D=8$. The simulated jet has dispersed as the profile has become flatter than the measured one, which is also seen in Fig. 6.6. The Reynolds stresses are rather isotropic. In the far field velocity field the inlet pipe BC has little effect. Considering the coarse mesh in the far field and the short sampling time, the cross stresses $\overline{u^{\prime} v^{\prime}} / U^{2}$ and $\overline{u^{\prime} w^{\prime}} / U^{2}$ are well predicted. 

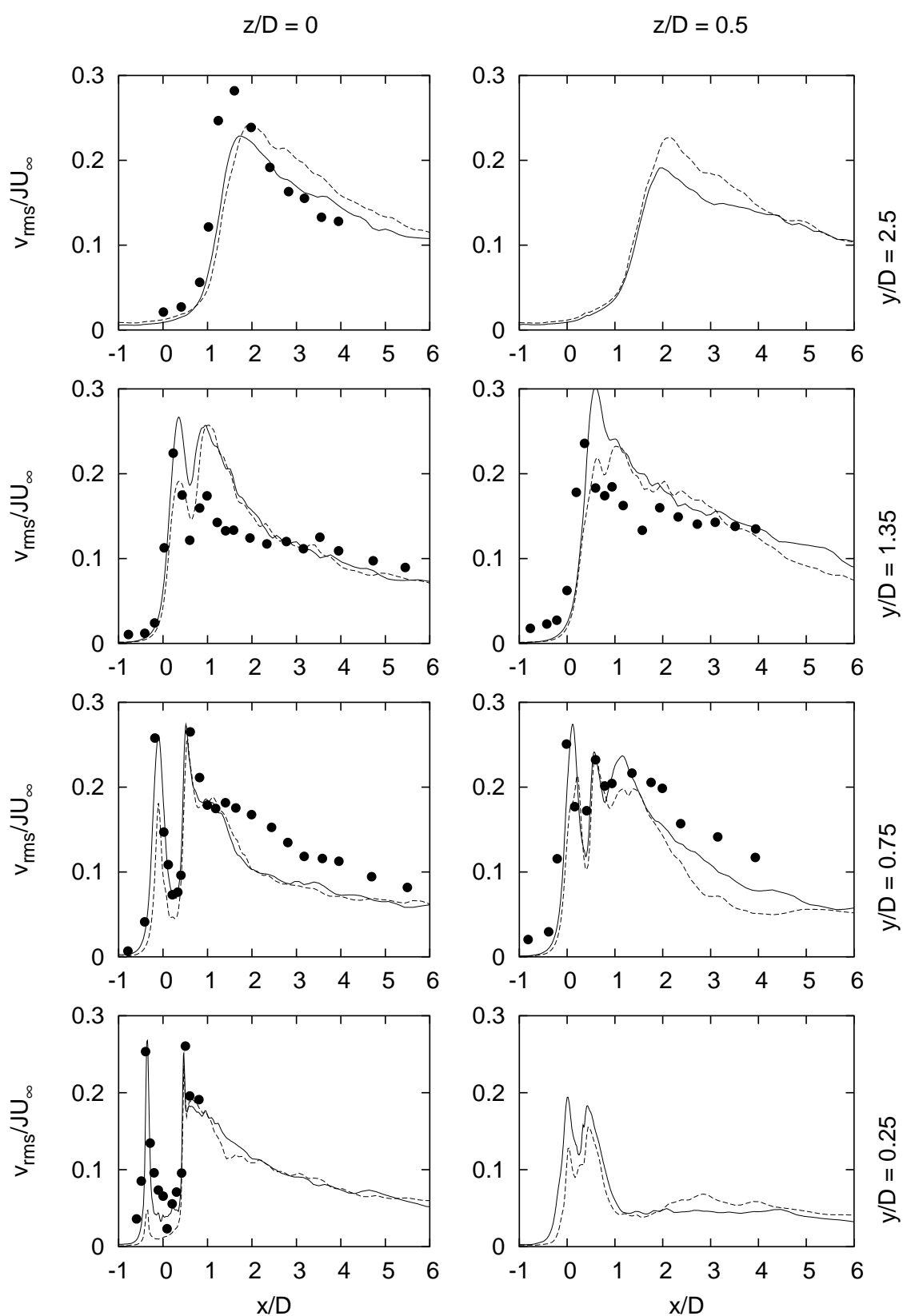

Figure 6.10: Wall-normal turbulence intensities plotted at various distances $y / D$ from the wall and $z / D$ from the central plane: $\operatorname{LESUBC}(-), \operatorname{LESSBC}(--)^{-}$, CDW $[6](\bullet)$. 

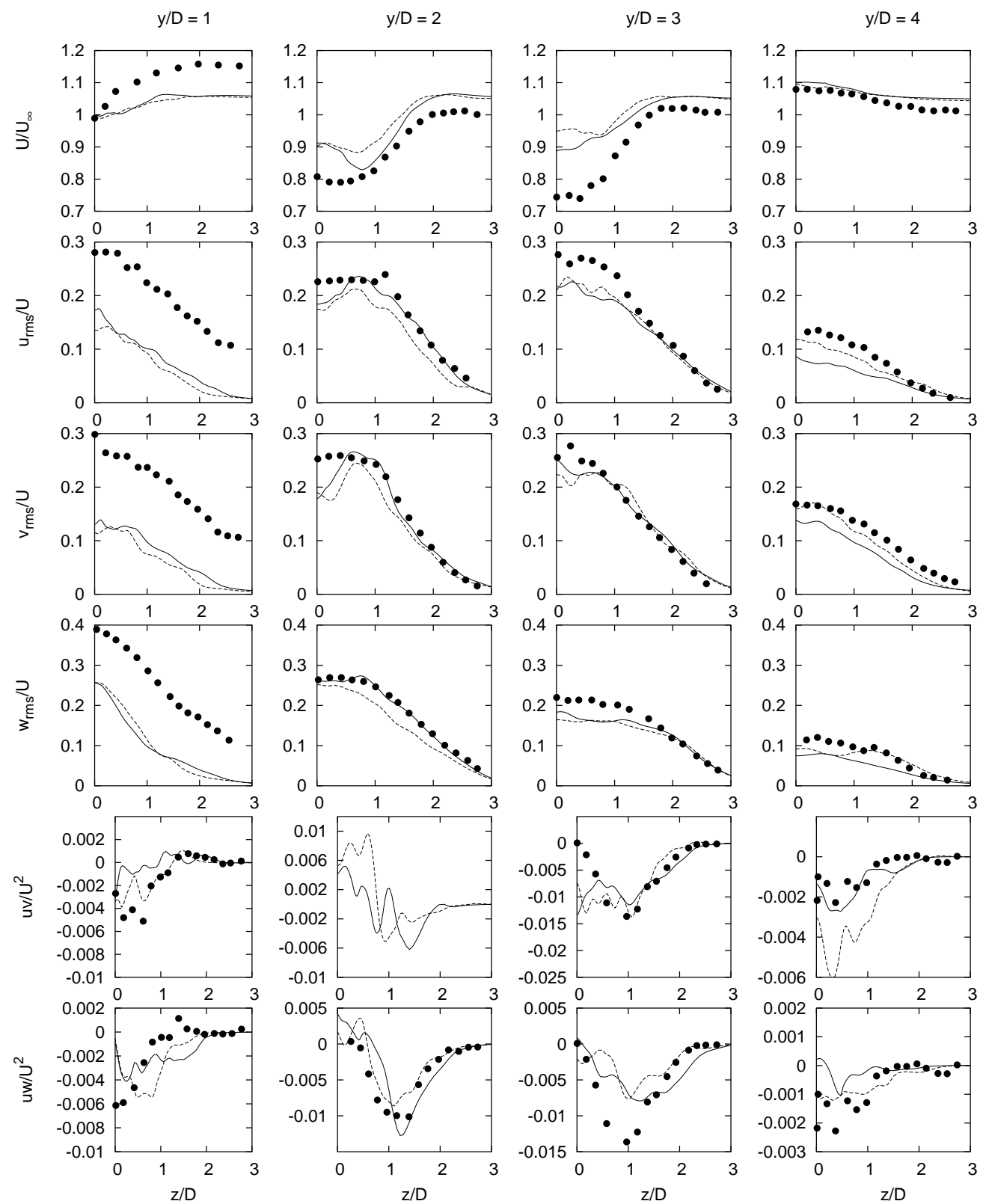

Figure 6.11: Mean velocity and Reynolds stress profiles at the position $x / D=8$ at various distances $y / D$ from the wall and $z / D$ from the central plane: LESUBC $(-), \operatorname{LESSBC}(--), \operatorname{CDW}[6](\bullet)$. 


\subsection{Scalar Mixing}

In a preliminary calculation with a lower Reynolds number it was observed that the central difference scheme led into spurious wiggles in the scalar field. The computed mixture fraction had values lower than zero and higher than one. This was observed also by Wille [39] who thought that using a TVD scheme would be unacceptable for excessive smearing. In the mean mixing fraction the spurious values were averaged off mostly. In preliminary tests a TVD-limited central difference for scalar in the convection term reduced overshoots effectively, but not completely. Here a MUSCL scheme is used with the minmod limiter Eq. (2.47). If the limiter is activated the discretization becomes upwind-biased and numerical dissipation is introduced. In the present case, a mixture fraction was restricted to lie between 0 and 1 , otherwise the calculation eventually diverged. This was the case both in the TVD-limited and the unlimited case. Fig. 6.12 shows the mean mixture fraction in the central plane. The TVD-limited scalar does not spread as much as the non-limited scalar. Both schemes underestimate the spreading under the jet. The non-limited scalar fluctuates more than the limited scalar, which explains the differences in the spreading. The schemes showed no clear difference in magnitudes for the stream-wise turbulent scalar transport. In other directions the transport terms were not recorded. Only a non-limited scalar discretization was used with LESUBC. The time evolving boundary in the jet pipe affects the scalar spreading even at the far field. The effect is stronger on the scalar field than on the velocity field. The observations above are confirmed by the contour plot in Fig. 6.13 in the far field plane at $x / D=8$. The TVD-limited scalar is shown on the right and the non-limited scalar of the LESSBC and the LESUBC in the centre. The measured contours of CDW are plotted with solid lines with the values next to them. Like the experiment, the LESUBC shows a separated region for $\theta / \theta_{J}=0.22$. Apparently the far field values are not converged completely as these regions are not symmetrically centered. Fig. 6.13 shows that in the flow the rich helium concentration does not coincide with the maximum velocity on the right. The flow with higher momentum 
thus originates from the free-stream fluid accelerated around the jet. The simulated velocity $U / U_{\infty}$ is flatter on the whole plane, as also seen in Fig 6.6. The LES contours are shown with dashed lines whose values lie in the range $0.9-1.04$. The smallest values are close to the bottom wall and in the centre plane, a value of 1.04 is detected also in the upper corner. The LESSBC and LESUBC velocity contours show no significant differences in this plot.
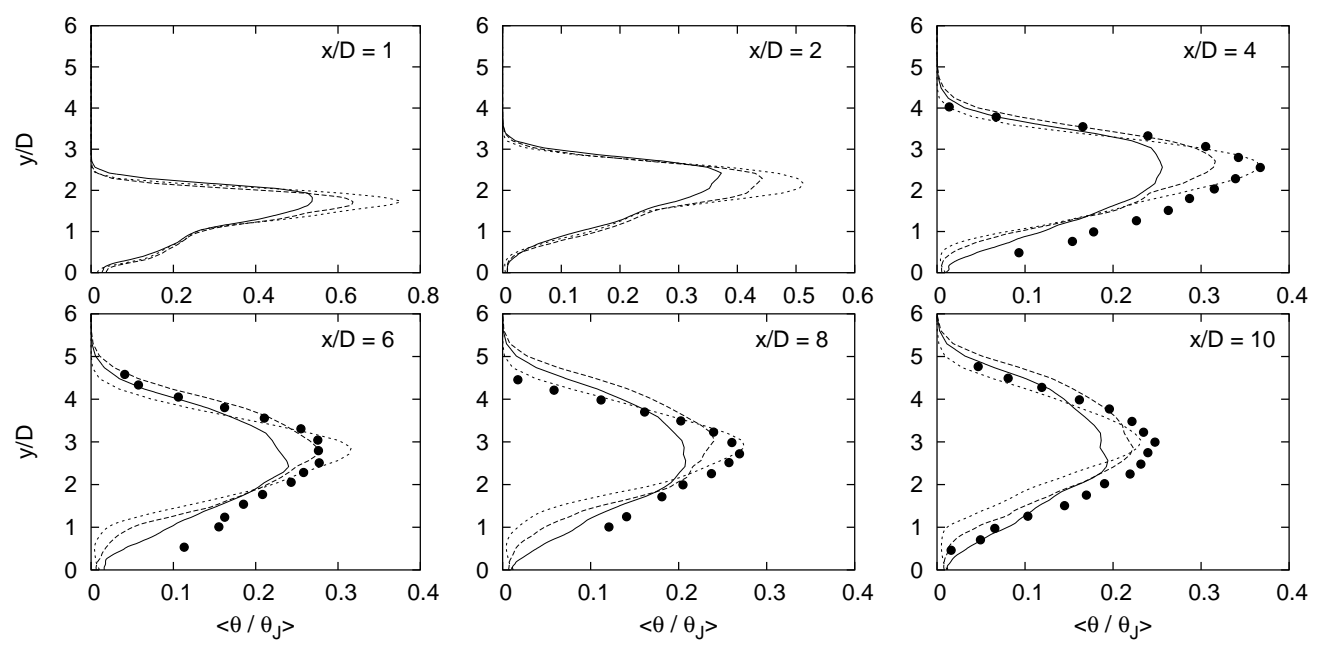

Figure 6.12: Mean mixture fraction in the central plane, $z / D=0$. LESUBC without any limiter $(-)$, LESSBC without any limiter (- - ), LESSBC with the minmod limiter $(\cdots)$, CDW $[6](\bullet)$. 

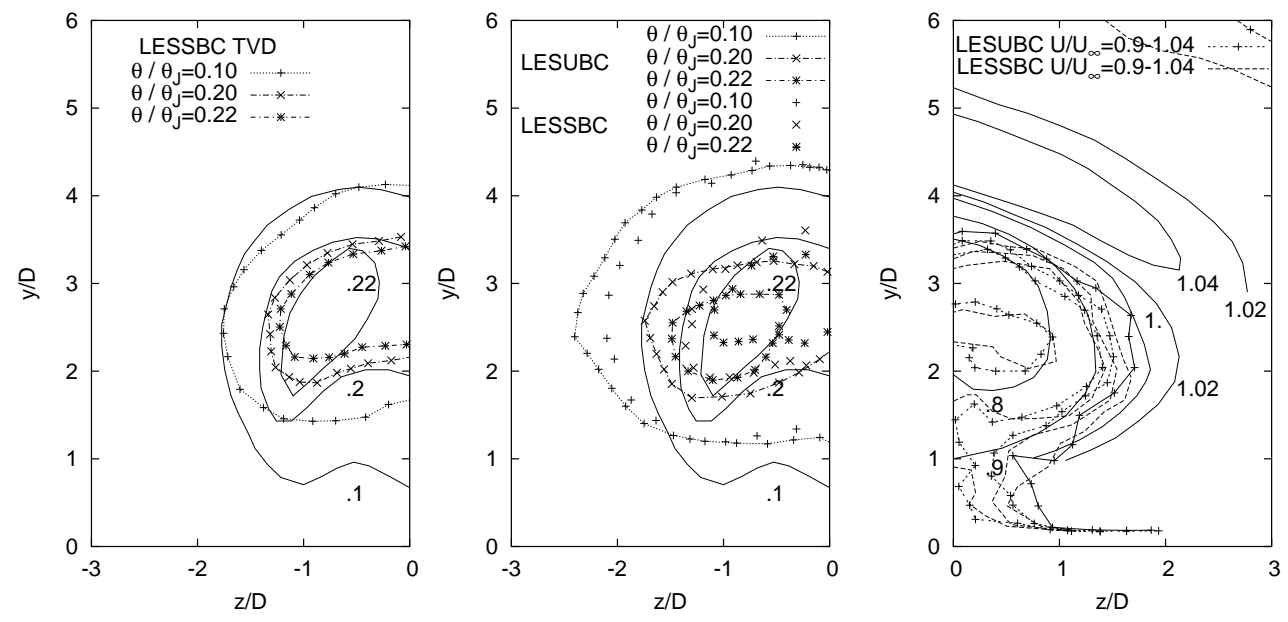

Figure 6.13: Contours of the mixture fraction and the mean stream-wise velocity in the plane, $x / D=8$. The TVD-limited scalar is on the left. In the centre the non-limited scalar and on the right the velocity with the LESUBC and LESSB. In all figures the solid line is the data from CDW [6]. 


\subsection{Conclusions}

The LES of a turbulent jet in a cross-flow with the present parameters has been performed with the steady-state and the unsteady boundary conditions. On the whole the differences between the cases are relatively small in this flow. The LES with the unsteady condition possesses a stronger back-flow in the lee of the jet where the cross-streamwise velocity profiles along the vertical lines are steeper. In the far field $(x / D \geq 8)$ the differences are small. The unsteady boundary increased the mixing of the scalar. The scalar was discretized with a TVD scheme in order to reduce the spurious wiggles, which consequently reduced also the mixing. In the preliminary computation with a smaller Reynolds number the minmod limiter with the second-order central difference did not completely remove the spurious values.

The resolution near the walls is probably too coarse for the present LES in which the flow is resolved to the wall with a no-slip boundary condition. A wall model or a hybrid RANS-LES model might be a good alternative if such a flow is computed with a similar resolution.

The LES reproduced many phenomena present in such a flow, like the shear layer ring vortices and the counter-rotating vortex pair. In general, a reasonable agreement with the measurements was obtained. The LES predicts an intense back-flow near the flat wall where no experimental data is available. It is possible that such a recirculation exists, but in any case the LES predicts it to be too high above the wall and probably too intense. 


\section{Concluding Remarks}

Turbulent simulations are often solved using explicit time stepping for best efficiency. In preliminary computation conducted in two-dimensional vortex-shedding flows this was the case too. At higher Reynolds numbers, where the difference in the cell sizes in the computational domain increases, the extra cost of the implicit solution is not so big any more. This is because the quality of the overall solution does not suffer even if somewhere in the domain Courant number exceeds unity. In complex domains the structured meshes often include small cells whose size is not determined by the flow physics but by the geometry. In such a case, limiting the Courant number smaller than one everywhere in the domain is not sensible.

Applying the Smagorinsky model or its dynamic derivative did not always improve the result in channel flow computations. If the computation includes any implicit diffusion and/or the Reynolds number is very low, the eddy viscosity produced by the sub-grid scale model may even deteriorate the solution. A numerical discretization error of second-order accuracy is greater by a decade or two in most of the domain in a channel flow. This applies to the stresses. At high Reynolds numbers and with an insufficient resolution the sub-grid scale model is needed to stabilize the computation via eddy viscosity.

The parallel solver developed showed a linear parallelization as equally sized domains were added to the computation. The localized dynamic model damped the eddy viscosity without any ad hoc damping functions and the unphysical need for the wall distance.

The large-eddy simulation of the jet in a cross-flow is a difficult case if compared to previous flows computed in this thesis. The flow possesses various features; the Reynolds number is higher and the periodic boundary conditions cannot be applied. Throughout this work the dynamic Smagorinsky model has not shown a lot of stabilizing features even if negative eddy viscosities are prevented. Therefore, the jet in cross-flow was computed using the traditional Smagorinsky model. The turbulent boundary conditions increased the spreading of the jet slightly, but in general the 
difference between the steady and the unsteady boundary conditions was quite small. The total variation diminished (TVD) scheme was used to discretize the scalar equation in order to remove non-physical oscillations. The TVD scheme reduced the wiggles but did not completely remove non-physical values. The TVD scheme reduced the spreading of the scalar. The LES reproduced many phenomena present in such a flow and a reasonable agreement with the measurements was obtained. The horseshoe vortex system around the exit near the wall was captured as well as a small separation of the flow within the pipe. The shear layer vortices and their evolution were present in an animation of the jet. The counter-rotating vortex pair evolves immediately after the jet exit which supports the idea that the vortex pair is initiated by the vorticity present in pipe.

The LES predicts an intense back-flow behind the jet near the flat wall where no experimental data is available. It is possible that such a recirculation exists, but in any case the resolution near the wall was probably not dense enough to calculate the correct friction with the present resolution.

The simulation of the jet in a cross-flow required an enormous amount of processor time. The computer power will have to increase vastly for LES to become practical for most engineering applications. In particular, the resolution of the boundary layer is expensive. Wall modelling or hybrid RANS-LES models will likely make timedependent and three-dimensional turbulence simulation closer to an engineering tool - at some time in the future. 


\section{References}

[1] J. Andreopoulos and W. Rodi. 1984. Experimental investigations of jets in a crossflow. Journal of Fluid Mechanics 138, pages 93-127.

[2] E. Balaras, C. Benocci, and U. Piomelli. 1995. Finite-Difference Computations of High Reynolds Number Flows Using the Dynamic Subgrid-Scale Model. Theoretical and Computational Fluid Dynamics 7, pages 207-216.

[3] T.T. Bui. 2000. A parallel, finite-volume algorithm for large-eddy simulation of turbulent flows. Computers \& Fluids 29, pages 877-915.

[4] R.L. Burden and J.D. Faires. 1993. Numerical Analysis, Fifth Edition. PWS Publishing Company. ISBN 0-534-93219-3.

[5] William Cabot and Parviz Moin. 1993. Large Eddy Simulation of Scalar Transport with the Dynamic Subgrid-Scale Model. In: Galperin B. and Orszag S.A. (editors), Large Eddy Simulation of Complex Engineering and Geophysical Flows, chapter 7, pages 141-158. Cambridge University Press, Cambridge, UK. ISBN 0-521-43009-7.

[6] D. Crabb, D.F.G Duráo, and J.H. Whitelaw. 1981. A Round Jet Normal to a Crossflow. Transactions of the ASME: Journal of Fluids Engineering 103, pages $568-580$.

[7] Simon Dahlström. 2003. Large Eddy Simulation of the Flow Around a HighLift Airfoil. Doctoral thesis, Chalmers University of Technology, Göteborg, Sweden.

[8] J.W. Deardorff. 1970. A numerical study of three-dimensional turbulent channel flow at large Reynolds numbers. Journal of Fluid Mechanics 41, pages 453-480. 
[9] J.M.J. den Toonder and F.T.M. Nieuwstadt. 1997. Reynolds number effects in a turbulent pipe flow for low to moderate Re. Physics of Fluids 9, pages 3398-3409.

[10] M. Germano, U. Piomelli, P. Moin, and W.H. Cabot. 1991. A dynamic subgridscale eddy viscosity model. Physics of Fluids A 7, pages 1760-1765.

[11] U. Ghia, K. Ghia, and C. Shin. 1982. High-Re Solutions for Incompressible Flow Using the Navier-Stokes Equations and a Multigrid Method. Journal of Computational Physics 48, pages 387-411.

[12] W. Gropp, E. Lusk, and A. Skjellum. 1999. Using MPI: Portable Parallel Programming with the Message-Passing Interface, Second Edition. The MIT Press, Cambridge, Massachusetts. ISBN 0-262-57132-3.

[13] J.O. Hinze. 1975. Turbulence. McGraw-Hill, New York, 2nd edition. ISBN 007-029037-7.

[14] Charles Hirsch. 1990. Computational Methods for Inviscid and Viscous Flows, volume 2 of Numerical Computation of Internal and External Flows. John Wiley \& Sons Ltd, Chichester. ISBN 0-471-92351-6.

[15] Jaakko Hoffren, Timo Siikonen, and Seppo Laine. 1995. Conservative Multiblock Navier-Stokes Solver for Arbitrarily Deforming Geometries. Journal of Aircraft 32 , no. 6, pages $1342-1350$.

[16] P. Johansson and Davidsson L. 1995. Modified Collocated SIMPLEC Algorithm Applied to Buoyancy-affected Turbulent Flow Using a Multigrid Procedure. Numerical Heat Transfer (Part B) 28, pages 39-57.

[17] J.F. Keffer and W.D. Baines. 1963. The round turbulent jet in a cross-wind. Journal of Fluid Mechanics 15, pages 481-497.

[18] R.M. Kelso, T.T. Lim, and A.E. Perry. 1996. An experimental study of round jets in cross-flow. Journal of Fluid Mechanics 306, pages 111-144. 
[19] J. Kim and P. Moin. 1985. Application of a Fractional-Step Method to Incompressible Navier-Stokes Equations. Journal of Computational Physics 59, pages $308-323$.

[20] J. Kim, P. Moin, and R. Moser. 1987. Turbulence Statistics in Fully Developed Channel Flow at Low Reynolds Number. Journal of Fluid Mechanics 177, pages $133-166$.

[21] M. Lesieur. 1997. Turbulence in Fluids, Third Revised and Enlargened Version. Kluwer Academic Publishers, Dordrecht. ISBN 0-7923-4416-2.

[22] D.K. Lilly. 1992. A proposed modification of the Germano subgrid-scale closure method. Physics of Fluids A 4, pages 633-635.

[23] P. Majander. 2000. Deveplopments in Large Eddy Simulation. Report 128, Helsinki University of Technology. ISBN 951-22-4861-1.

[24] A. Miettinen. 1997. A Study of the Pressure Correction Approach in the Colocated Grid Arrangement. Technical report no. 110, Helsinki University of Technology, Laboratory of Applied Thermodynamics. ISSN 1237-8372.

[25] F.M. Najjar and D.K. Tafti. 1996. Evaluation of of the Dynamic SubgridScale Stress Model in Finite Differenced LES: Effects of Grid Resolution and Inhomogeneous Test Filttering. In: Symposium on LES and DNS of Turbulent Flow, pages 229-236. Atlanta. FED-Vol. 242.

[26] F.M. Najjar and D.K. Tafti. 1996. Study of discrete test filters and finite difference approximations for the dynamic subgrid-scale stress model. Physics of Fluids 8, no. 4, pages 1076-1088.

[27] S.D. Peterson and M.W. Plesniak. 2004. Evolution of jets emanating from short holes into crossflow. Journal of Fluid Mechanics 503, pages 57-91.

[28] U. Piomelli and J. Liu. 1995. Large-eddy simulation of rotating channel flows using a localized dynamic model. Physics of Fluids 7, no. 4, pages 839-848. 
[29] A.K. Prasad and J.R. Koseff. 1989. Reynolds number and end-wall effects on a lid-driven cavity flow. Physics of Fluids A 1, no. 2, pages 208-218.

[30] C.M. Rhie and W.L. Chow. 1983. Numerical Study of the Turbulent Flow Past an Airfoil with Trailing Edge Separation. AIAA Journal 21, no. 11, pages $1525-1532$.

[31] A. Rizzi, P. Eliasson, I. Lindblad, C. Hirsch, C. Lacor, and J. Haeuser. 1993. The Engineering of Multiblock/Multigrid Software for Navier-Stokes Flows on Structured Meshes. Computers \& Fluids 22, no. 2, pages 341-367.

[32] P. Sagaut, E. Montreuil, and O. Labbé. 1999. Assessment of some self-adaptive SGS models for wall bounded flows. Aerospace Science and Technology 3, no. 6 , pages $335-344$.

[33] J. Smagorinsky. 1963. General circulation experiments with the primitive equations, Part I: The basic experiment. Montly Weather Review 91, pages 99-152.

[34] H. Tennekes and J.L. Lumley. 1972. A First Course in Turbulence. The MIT Press, Cambridge, Massachusetts, 1st edition. ISBN 0-262-20019-8.

[35] B. Vreman, B. Geurts, and H. Kuerten. 1994. Discretization error dominance over subgrid terms in large eddy simulation of compressible shear layers in 2D. Communications in Numerical Methods in Engineering 10, pages 785-790.

[36] B. Vreman, B. Geurts, and H. Kuerten. 1995. A priori tests of large eddy simulation of the compressible plane mixing layer. Journal of Engineering Mathematics 29, pages 299-327.

[37] B. Wegner, Y. Huai, and Sadiki A. 2004. Comparative study of turbulent mixing in jet in cross-flow configurations using LES. International Journal of Heat and Fluid Flow 25, pages 767-775. 
[38] Frank M. White. 1991. Viscous Fluid Flow. McGraw-Hill Book Co., New York, 2nd edition. ISBN 0-07-069712-4.

[39] M. Wille. 1997. Large Eddy Simulation of jets in cross flows. Ph.d. thesis, Imperial College of Science, Technology and Medicine, Department of Chemical Engineering, London.

[40] L.L Yuan, R.L. Street, and J.H Ferziger. 1999. Large-eddy simulations of a round jet in crossflow. Journal of Fluid Mechanics 379, pages 71-104. 
ISBN-13 978-951-22-8404-7

ISBN-10 951-22-8404-9

ISBN-13 978-951-22-8405-4 (PDF)

ISBN-10 951-22-8405-7 (PDF)

ISSN 1795-2239

ISSN 1795-4584 (PDF) 